\title{
Control-oriented modeling of two-stroke diesel engines with exhaust gas recirculation for marine applications
}

Xavier Llamas and Lars Eriksson

The self-archived postprint version of this journal article is available at Linköping University Institutional Repository (DiVA):

http://urn.kb.se/resolve?urn=urn:nbn:se:liu:diva-147828

N.B.: When citing this work, cite the original publication.

Llamas, X., Eriksson, L., (2018), Control-oriented modeling of two-stroke diesel engines with exhaust gas recirculation for marine applications, Journal of Engineering for the Maritime Environment (Part M). https://doi.org/10.1177/1475090218768992

Original publication available at:

https://doi.org/10.1177/1475090218768992

Copyright: SAGE Publications (UK and US)

http://www.uk.sagepub.com/home.nav 


\title{
Control-oriented modeling of two-stroke diesel engines with EGR for marine applications
}

\author{
Xavier Llamas ${ }^{1}$ and Lars Eriksson ${ }^{1}$
}

\begin{abstract}
Large marine two-stroke diesel engines are widely used as propulsion systems for shipping worldwide and are facing stricter $N O_{x}$ emission limits. Exhaust gas recirculation (EGR) is introduced to these engines to reduce the produced combustion $N O_{x}$ to the allowed levels. Since the current number of engines built with EGR is low and engine testing is very expensive, a powerful alternative for developing EGR controllers for such engines is to use control-oriented simulation models. Unfortunately, the same reasons that motivate the use of simulation models also hinder the capacity to obtain sufficient measurement data at different operating points for developing the models. A Mean Value Engine Model (MVEM) of a large two-stroke diesel with EGR that can be simulated faster than real time is presented and validated. An analytic model for the cylinder pressure that captures the effects of changes in the fuel control inputs is also developed and validated with cylinder pressure measurements. A parameterization procedure that deals with the low number of measurement data available is proposed. After the parameterization, the model is shown to capture the stationary operation of the real engine well. The transient prediction capability of the model is also considered satisfactory which is important if the model is to be used for EGR controller development during transients. Furthermore, the experience gathered while developing the model about essential signals to be measured is summarized, which can be very helpful for future applications of the model. Finally, models for the ship propeller and resistance are also investigated, showing good agreement with the measured ship sailing signals during maneuvers. These models give a complete vessel model and make it possible to simulate various maneuvering scenarios, giving different loading profiles that can be used to investigate the performance of EGR and other controllers during transients.
\end{abstract}

\section{Keywords}

Mean Value Engine Model, Dynamic Simulation, Parameterization, Exhaust Gas Recirculation, Ship Propulsion

\section{Introduction}

Over the last year, maritime transport growth slowed down. However, shipping is still growing, and for the first time the estimated world seaborne trade volume surpassed 10 billion tons. ${ }^{1}$ The required technical development to achieve an overall clean and efficient transportation in our society has to involve the marine sector as well. The regulations that have driven the automotive industry started several decades ago, while the regulations affecting marine diesels began at the beginning of the last decade. Hence, the process of reducing the environmental impact of the shipping industry is ongoing. The International Maritime Organization has developed the stricter Tier III emission limits ${ }^{2}$ on $\mathrm{NO}_{\mathrm{x}}$, for new vessels built after January 2016.

Low-speed two-stroke diesel engines usually propel the largest vessels, e.g., tankers, bulk carriers, and container ships. These low-speed engines have high fuel efficiency, however, they are also responsible for large amounts of pollutant emissions, like $\mathrm{NO}_{\mathrm{x}}$ and $\mathrm{SO}_{\mathrm{x}}$. The large reduction in $\mathrm{NO}_{\mathrm{x}}$ emissions enforced by the Tier III compared to the previous Tier II regulations cannot be fulfilled by only improving the combustion of these engines. Thus, new technologies are being developed in order to attain the emission reductions while still keeping a good specific fuel oil consumption (SFOC), which is a good indication of $\mathrm{CO}_{2}$ emissions. The two most common technical solutions are Selective Catalytic Reduction (SCR) and Exhaust Gas Recirculation, (EGR). SCR is an after-treatment technology

\footnotetext{
${ }^{1}$ Linköping University, Sweden

Corresponding author:

Xavier Llamas, Vehicular Systems, Department of Electrical Engineering, Linköping University, Linköping SE-581 83, Sweden

Email: xavier.llamas.comellas@liu.se
} 
that removes the formed $\mathrm{NO}_{\mathrm{x}}$ during the combustion. On the other hand, EGR reduces the formation of $\mathrm{NO}_{\mathrm{x}}$ during the combustion. This is achieved by recirculating a fraction of the burned gas back to the engine scavenging manifold. As a result, the heat capacity of the gas that enters the cylinders is increased and thus the peak temperatures are reduced. The model presented here is dedicated to the EGR application only.

The Tier III limits are only enforced in certain $\mathrm{NO}_{\mathrm{x}}$ Emission Control Areas (NECAs), e.g., the North American coastal area. Currently, the number of NECAs is low but it will be increased in the coming years. This implies that the target $\mathrm{NO}_{\mathrm{x}}$ reduction has to be achieved when the ship is maneuvering to enter a harbor. This is especially challenging for the EGR control system since rapid transient accelerations required during maneuvering can conflict with the desire to keep a high EGR flow rate. The low oxygen concentration in the combustion chamber limits the amount of fuel that can be burned without resulting in incomplete combustion that produces undesired black smoke. The transient load increase is currently the main challenge for the EGR controller, and to improve its performance, transient EGR testing is required.

In the automotive industry, EGR can be widely tested with the help of well-equipped engine test beds. For the case of large two-stroke engines, the number of available test beds is much lower. Mainly because of the lower number of manufacturers as well as the higher cost of building test beds for large two-stroke engines. Even if the main engine designers may have a test bed for research and development, e.g., the test engine at MAN Diesel \& Turbo research center, the high running cost and the low time availability limits the number of engine tests that can be carried out. Another option for EGR testing is to perform it on engines with EGR system installed on currently sailing ships. This possibility is, of course, limited to agreements with the owners and time availability at sea. Moreover, since EGR has just been recently introduced, there are not many ships equipped with an EGR system available. Besides the low number of EGR engines to perform tests on, the high cost of running these large engines is also an important factor that limits the testing possibilities. Hence, in order to overcome these limitations, a dynamic simulation model of such engine is a handy tool for EGR controller development. Unfortunately, the limited availability of real engine testing measurements has also a negative impact on the available data quality and quantity to be used for engine model development and validation. Further discussions about how to deal with the low data availability are included in this article, based on the experience gained during the complete model development.

The main objective of this study is to present and validate a dynamic model for a large two-stroke diesel engine with EGR. The proposed model follows the modeling principles of Mean Value Engine Models (MVEMs), widely used for automotive engine control research. Some advantages of such models are the low computational complexity and the capacity to capture the dominant system dynamics. A general overview of MVEMs and their applications can be found in Eriksson and Nielsen. ${ }^{3}$ In addition, ship resistance and propeller models are included in order to study the dynamic interactions between the engine and the ship. The model's main application is for EGR and fuel controller development, e.g., Nielsen et al. , ${ }^{4,5}$ but it can also be a valuable tool for sizing engine components and for optimizing and benchmarking different engine architectures during the design phase.

\subsection{Related Research}

Internal combustion engine modeling and control has been an important research topic over the last decades, and a large amount of research has been carried out concerning MVEM development, especially for automotive applications. An important work that lays the foundation for such models is Heywood. ${ }^{6}$

Engine models for large marine diesel engines have not been as widely studied as for the automotive case. Large marine diesels can be four or two-stroke based engines, where two-stroke is the standard choice for the highest power outputs. During the eighties several authors started to model the dynamic behavior of large two-stroke diesel engines, the main focus was the shaft speed dynamics to improve the speed governors. ${ }^{7,8}$ A special mention is given to the work presented in Hendricks, ${ }^{9}$ where the MVEM term was coined. More recently developed MVEMs of such engines are presented in Xiros ${ }^{10,11}$ and Theotokatos. ${ }^{12}$ A model for low load operation is presented in Guan et al., ${ }^{13}$ where it is shown that a compressor model capable of extrapolating to low load is required and proposed. Further development was done in the two-stroke model presented in Guan et al., ${ }^{14}$ where a zero-dimensional crank angle resolved cylinder pressure model is implemented. Simulations of this latter zero-dimensional model are used in Theotokatos et al. ${ }^{15}$ to define extension functions to certain engine parameters. This results in a computationally fast MVEM that captures the effects of changes in the combustion input signals. For the marine four-stroke case, some examples are Malkhede et al. ${ }^{16}$ and Baldi et al. ${ }^{17}$

Since EGR is a rather new system applied to twostroke engines, not much research has been carried out regarding EGR engine modeling. On the other hand, EGR modeling has been much more investigated for the automotive-size combustion engines. Many ideas can be adapted from this field to the two-stroke case. However, fundamental differences exist between four and two-stroke engines, which introduce new modeling challenges. The most important is that the scavenging manifold pressure 
in two-stroke diesels needs to be higher than the exhaust manifold pressure. Hence, the EGR flow has to be forced by means of a blower.

One of the first attempts to model two-stroke engines with EGR is Hansen et al., ${ }^{18}$ where the work carried out in Wahlström and Eriksson ${ }^{19}$ was adapted from the fourstroke to the two-stroke application. Further modeling work was done in Alegret et al. ${ }^{20}$ and extended for low load simulation in Llamas and Eriksson. ${ }^{21}$ All three mentioned publications had the 4T50ME-X test engine as modeling object. More recent work is presented in Nielsen et. al., ${ }^{22}$ which introduces a model reduction that captures the main system dynamics and is suitable to use for an EGR controller. In this latter publication, the modeling approach is tested for two different engines, the 4T50ME-X test engine and a 6S80ME-C9.2 engine installed in a container ship. In Wang et al., ${ }^{23,24}$ the commercial software GTpower is used to develop one-dimensional models of twostroke engines with EGR. The effects of exhaust gas bypass (EGB) and cylinder bypass (CB) on EGR operation are investigated using the developed model.

\subsection{Contributions}

A complete control oriented MVEM of a marine large twostroke diesel engine with EGR is described. New model components are investigated, in particular, an analytic cylinder pressure model that can capture the influence of changes in the cylinder pressure control signals, e.g., injection angle and exhaust valve opening and closing. The cylinder model is developed using cylinder pressure measurements from a container ship engine. Moreover, a model for the heat transfer in the exhaust is proposed to improve the exhaust temperature predictions compared to previous modeling work.

A parameterization process, designed to deal with the scarcity of measurement data is also described. It is shown to obtain good model parameters that are capable of matching the measured operating signals of the engine for a wide range of loads and engine running modes (ERM). Furthermore, the experience gathered through the complete development of this engine model is used to identify important measurement signals that are currently unavailable but could be included in future measurement campaigns. These identified signals can be valuable to the research community when parameterizing the proposed model to other engines, and for further validation of the engine component models.

In addition, propeller and ship resistance models are investigated. Together with the proposed engine model, they constitute a complete simulation model of a container ship. The model is ready to be used for simulationbased investigations of EGR controller performance during typical load transients introduced when the vessel is maneuvering.

\subsection{Outline}

The experimental data used to develop, parameterize and validate the proposed model is introduced in Section 2. Section 3 presents the modeling approach of the different components, with special attention to the proposed analytical cylinder pressure model. Section 4 describes the model parameterization procedure. Section 5 contains the model validation against the available measurements for both stationary and dynamic conditions. A short discussion about guidelines for future engine testing is included in Section 6. Section 7 introduces the propeller and ship resistance models used to simulate the complete container ship and shows a simulation example to validate the model.

\section{Experimental Data}

The measurement data used to design and tune the proposed MVEM is taken from the different sensors mounted on the ship engine. The sensors record the different engine operating signals while the ship is sailing. Then, the data is post-processed to extract stationary points for the model tuning and validation procedures. The post-processing is also done to avoid having a repetition of operating points, which could bias the model accuracy towards the operating region with the higher number of points. The measured engine operation spans a reasonably wide range of load and operating modes. A total of 52 stationary points have been extracted for the parameterization process. However, the amount of different operating points collected is not close to the usual engine mapping done for calibration of automotive MVEMs. Nevertheless, the available data is sufficient to parameterize the proposed model as will be shown in Section 5. In addition, 40 stationary points are reserved for the stationary validation of the engine model.

Furthermore, in-cylinder pressure measurements are available for 24 different operating points. These were synchronized with the rest of the measuring setup system to gather data for developing the new cylinder model described in Section 3.7. All in-cylinder pressure measurements were recorded earlier than the data used for stationary point extraction. Therefore, the cylinder measurements cannot be synchronized with the previously mentioned extracted stationary points. Moreover, the corresponding operating points to each in-cylinder pressure measurement were not long enough to obtain a stabilized exhaust temperature measurement, so this data has only been used for developing the cylinder pressure model.

For future applications, the stationary shop test data collected during the engine commissioning should be sufficient to parameterize the proposed model. This is 
important since the engine model could be tuned before it is installed on the ship. In this case, this was not possible since certain signals required were not available, e.g., the cylinder exhaust valve opening and closing angles.

\section{Engine Modeling}

The modeled engine is a MAN Diesel \& Turbo 6S80MEC9.2 two-stroke uniflow diesel engine equipped with an EGR system for Tier III operation. It has six cylinders with $0.8 \mathrm{~m}$ bore and $3.45 \mathrm{~m}$ stroke, and it can deliver a maximum rated power of $23 \mathrm{MW}$ at $73.9 \mathrm{rpm}$. The main characteristics of the engine air path can be seen in Figure 1. The engine has two turbochargers in parallel that deliver the necessary air. The EGR system takes gas from the exhaust manifold and delivers it to the gas mixer using two EGR blowers.

The control volumes are depicted as rectangles, with the corresponding model states written inside. The generic control volume has states for pressure, temperature, and gas concentration. However, not all control volumes contain all state dynamics of the generic model, as can be seen in Figure 1. The three control volumes in the EGR loop assume that the volume is isothermal. Thus, its temperature is set by the inlet flows temperature. The control volume of the turbine outlet assumes that the gas concentration is equal to the inlet gas concentration. Finally, the compressor inlet and outlet control volumes assume that the only dynamic change occurs with the pressure. Moreover, the turbocharger speeds and the exhaust manifold wall temperature are also model states as will be discussed in Sections 3.3 and 3.10.

In total, the MVEM has 41 states and 11 control inputs, shown in Figure 1. Four control inputs correspond to the cylinder model; $\alpha_{E V C}, \alpha_{E V O}, \alpha_{i n j}$ and $Y$. Four more control inputs are required for the EGR system; $u_{S D V}$, $u_{C O V}, \omega_{e b, 1}$, and $\omega_{e b, 2}$. Finally, three more general control inputs are needed; $u_{t c}, u_{E G B}$ and $u_{A u x}$. The air ambient pressure, temperature and relative humidity can be either taken as constants or set equal to the measured signals.

The engine has four main Engine Running Modes (ERM), which are included in the proposed model. Number one is with both turbochargers running and no EGR operation. The second mode is with only the main turbocharger running, using the cut-out valve $u_{t c}$, and no EGR operation. Number 3 is low EGR operation with only the main turbocharger and one of the two EGR blowers operating. Finally, the fourth mode is high EGR flow with both EGR blowers and only the main turbocharger running.

\subsection{Gas Composition and Properties}

When recirculating burned gases, the amount of oxygen in the scavenge gas is reduced. This increases the heat capacity

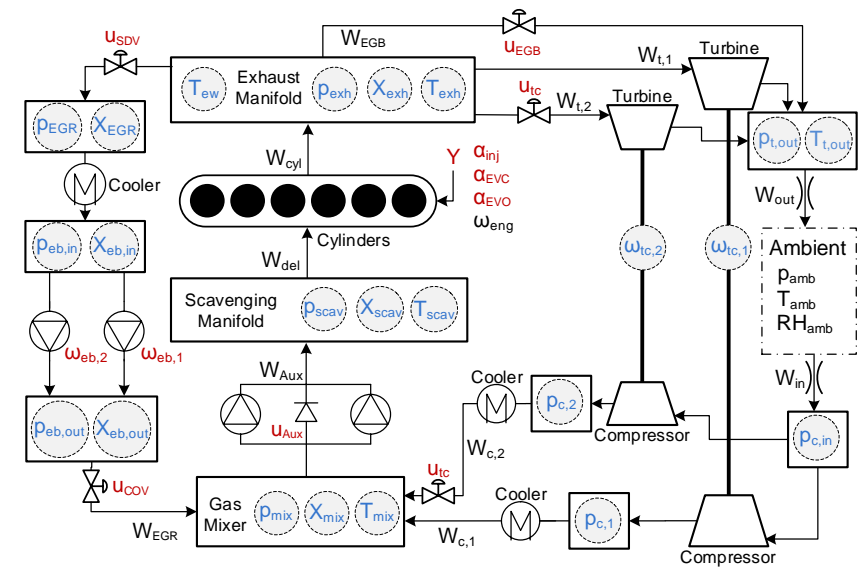

Figure 1. Diagram of the modeled two-stroke engine with the model states inside the depicted control volumes. The diagram also contains the location of the different control signals and general mass flows.

of the gas and thus reduces the amount of $N O_{x}$ produced during combustion. However, EGR has the undesired effect of lowering the amount of fuel that can be burned since this is directly proportional to the amount of oxygen in the combustion chamber.

Keeping track of the scavenging gas composition is crucial in a diesel engine with EGR. Thus, the engine working fluid is modeled as a mixture of $\mathrm{O}_{2}, \mathrm{CO}_{2}, \mathrm{H}_{2} \mathrm{O}$, $\mathrm{SO} 2$ and $\mathrm{N}_{2}$. The reason to include more species than $\mathrm{O}_{2}$ is to make the model capable of working with different kind of fuels, with more or less $\mathrm{SO}_{2}$ content. Note that only lean operation is considered, and thus there is no need to consider $\mathrm{CO}$ and $\mathrm{H}_{2}$. Moreover, since the scavenging oxygen sensor is measuring molar concentrations, keeping track of the complete gas composition simplifies the process to change from mass to molar concentrations and viceversa. The concentration vector in an arbitrary control volume is defined as

$$
X=\frac{\left[m_{\mathrm{O}_{2}}, m_{\mathrm{CO}_{2}}, m_{\mathrm{H}_{2} \mathrm{O}}, m_{\mathrm{SO}_{2}}\right]^{T}}{m_{\text {tot }}}
$$

$N_{2}$ is not included in the concentration vector since it can be computed as the remaining part. To ensure that the mass conservation law is not violated, the mass flow of $N_{2}$ entering and exiting the engine model can be monitored to validate the numerical integrations during simulations. The gas thermodynamical properties are calculated using the NASA polynomials, ${ }^{25}$ and assuming a thermally perfect gas, i.e., the heat capacities of the gases are functions of temperature and composition but not pressure. The inlet fresh air concentration is calculated with the standard air oxygen concentration and the measured relative humidity $(\mathrm{RH})$ at the inlet. 


\subsection{Control Volumes}

In an MVEM, the different pipe volumes of the engine are seen as control volumes that store mass and energy. The filling and emptying of those volumes define the dynamic behavior of the model. Thus, the bigger the volume is, the slower it will be to change its current states. The largest control volumes in a two-stroke engine are the scavenging and exhaust manifolds and these define the main time constants together with the turbocharger rotational dynamics. In addition, several others are added in order to avoid algebraic loops that are introduced when connecting several flow restrictions in series, e.g., the control volumes at the compressor outlets.

For the main control volumes, mixing, scavenging, and exhaust, mass and energy conservation laws are used to derive the state differential equations. Note that the gas thermodynamic properties are a function of the gas concentration and temperature, i.e., $c_{v}(X, T)$ and $R(X)$. Starting from the conservation of mass as it is done in Eriksson and Nielsen, ${ }^{3}$ the change in stored mass in the control volume can be written as

$$
\frac{d m}{d t}=\sum_{j} W_{i n, j}-\sum_{k} W_{o u t, k}
$$

Considering the control volume as a well-stirred mixer, the dynamics of the concentration states can be derived starting with the conservation of mass equation, (2), which yields

$$
\frac{d X}{d t}=\frac{R(X) T}{p V} \sum_{j}\left(X_{i n, j}-X\right) W_{i n, j}
$$

Applying the conservation of energy law will define the differential equation governing the temperature change in the control volume. This derivation is done starting with the same equation as in Eriksson and Nielsen. ${ }^{3}$ However, in this case the specific heats are not considered as constants which makes the expressions longer. After rearranging the terms it can be written as

$$
\begin{aligned}
\frac{d T}{d t} & =\frac{\frac{R(X) T}{p V}(A-B)-T\left(\frac{\partial c_{v}(X, T)}{\partial X}\right)^{\mathrm{T}} \frac{d X}{d t}}{c_{v}(X, T)+T \frac{\partial c_{v}(X, T)}{\partial T}} \\
A & =\sum_{j} W_{i n, j}\left(c_{v}\left(T_{i n, j}, X_{i n, j}\right)+R\left(X_{i n, j}\right)\right) T_{i n, j} \\
B & =\sum_{k} W_{\text {out }, k} R(X) T+\sum_{j} W_{i n, j} c_{v}(X, T) T+\dot{Q}
\end{aligned}
$$

where $\dot{Q}$ represents the heat transfer from the control volume to the surrounding. Note that the gas concentration derivative, $\frac{d X}{d t}$, has to be inserted in (4a).
Having the control volume temperature and stored mass as states, the pressure in the control volume can be computed using the ideal gas law. This results in a compact notation but requires an extra nonlinear equation to obtain the pressure as the model output. Another approach, which is the one followed here, is to get the pressure as a state in the control volume and thus directly available as a model output. By differentiating the ideal gas law, the pressure dynamics can be written as

$$
\frac{d p}{d t}=\frac{R(X) T}{V} \frac{d m}{d t}+\frac{p}{R(X)}\left(\frac{d R(X)}{d X}\right)^{\mathrm{T}} \frac{d X}{d t}+\frac{p}{T} \frac{d T}{d t}
$$

with the concentration and temperature derivatives, $\left(\frac{d X}{d t}, \frac{d T}{d t}\right)$, directly substituted in (5). Note that the mass derivative, $\frac{d m}{d t}$, appears in (5) for compact notation. But it does not need to be integrated since it only represents the difference between entering and exiting mass flows to the control volume (2). Appendix A contains information about how to compute the thermodynamical properties of the gas. Moreover, it also contains definitions of the derivatives appearing in the previous expressions.

The previous equations are written for the generic control volume case. However, some of the control volumes in the model are simplified, neglecting the temperature or the gas concentration dynamics. This is shown in Figure 1, where the states are shown in each control volume. The simplifications are done by setting the corresponding derivatives to zero in the previous derived generic equations. Note that the heat transfer in the Gas mixer and Scavenging manifold are set to zero, while the heat transfer model in the exhaust manifold is described in Section 3.10.

\subsection{Turbochargers}

The engine has two turbocharging units working in parallel to supply the engine with sufficient air mass flow. When the engine operates with EGR the secondary turbocharger is disabled with the use of the cut-out valves, $u_{t c}$.

The compressor model structure is defined as

$$
\begin{aligned}
W_{c} & =f_{W_{c}}\left(p_{c, \text { in }}, p_{c, \text { out }}, T_{c, \text { in }}, \omega_{t c}\right) \\
\eta_{c} & =f_{\eta_{c}}\left(p_{c, \text { in }}, p_{c, \text { out }}, T_{c, \text { in }}, \omega_{t c}\right) \\
T_{c, \text { out }} & =T_{c, \text { in }}+\frac{T_{c, \text { in }}}{\eta_{c}}\left\{\left(\frac{p_{c, \text { out }}}{p_{c, \text { in }}}\right)^{\frac{\gamma_{c}-1}{\gamma_{c}}}-1\right\} \\
P_{c} & =W_{c} c_{p, c}\left(T_{c, \text { out }}-T_{c, \text { in }}\right)
\end{aligned}
$$

where the low load extrapolation capable compressor model described in Llamas and Eriksson ${ }^{26,27}$ is inserted in (6a) and (6b). This compressor model has been proven to work well for low load simulation. ${ }^{21}$ 
The turbine model outputs are computed as

$$
\begin{aligned}
W_{t} & =f_{W_{t}}\left(p_{t, \text { in }}, p_{t, \text { out }}, T_{t, \text { in }}, \omega_{t c}\right) \\
\eta_{t} & =f_{\eta_{t}}\left(p_{t, \text { in }}, p_{t, \text { out }}, T_{t, \text { in }}, \omega_{t c}\right) \\
T_{t, \text { out }} & =T_{t, \text { in }}-\eta_{\mathrm{t}} T_{t, \text { in }}\left\{1-\left(\frac{p_{t, \text { out }}}{p_{t, \text { in }}}\right)^{\frac{\gamma_{t}-1}{\gamma_{t}}}\right\} \\
P_{t} & =W_{t} c_{p, t}\left(T_{t, \text { in }}-T_{t, \text { out }}\right)
\end{aligned}
$$

where the turbine mass flow and efficiency models from Llamas and Eriksson ${ }^{21}$ are inserted in (7a) and (7b).

The shaft speeds of both turbochargers are model states, defined by the power balance between each turbine and compressor. The turbocharger speeds are the slowest dynamics of the model and thus the most dominant states for the engine transient behavior. Using the formulation described in Eriksson and Nielsen, ${ }^{3}$ each shaft speed is computed as

$$
\frac{d}{d t} \omega_{t c}=\frac{P_{t} \eta_{m e c h}-P_{c}}{J_{t c} \omega_{t c}}
$$

where $\eta_{\text {mech }}$ describes the friction losses of the connecting shaft and it is used as a tuning parameter.

\subsection{Air Coolers Restrictions and Valves}

All valves in the model, see Figure 1, are modeled as compressible turbulent restrictions with the valve opening angle as input. ${ }^{3}$

$W_{v}=\frac{A_{e f f}\left(u_{v}\right) p_{v, i n}}{\sqrt{R_{v, \text { in }} T_{v, i n}}} \sqrt{\frac{2 \gamma_{v, i n}}{\gamma_{v, \text { in }}-1}\left(\Pi^{\frac{2}{\gamma_{v, i n}}}-\Pi^{\frac{\gamma_{v, i n}+1}{\gamma_{v, i n}}}\right)}$

$T_{v, \text { out }}=T_{v, \text { in }}$

The relation between the effective area, $A_{e f f}$, and the valve opening signal, $u_{v}$, is highly nonlinear. The engine designer identified this nonlinear relation and provided it to the authors. The maximum effective area for each valve used to scale the static relation, is considered a tuning parameter; $A_{S D V}, A_{C O V}, A_{E G B}$. The turbocharger cut-out valve, controlled with $u_{t c}$, could be modeled as a compressible restriction. However, in order to avoid having to include more tuning parameters and control volumes, instead, it is modeled as a switch that enables or disables flow through the secondary turbocharger.

All air coolers consist of a flow restriction, a cooling element, and a water mist catcher (WMC). The restriction is modeled as an incompressible turbulent restriction, ${ }^{3}$

$$
\begin{aligned}
& W_{a c}=A_{a c} \sqrt{\frac{p_{a c, \text { in }}}{R_{a c, \text { in }} T_{a c, \text { in }}}\left(p_{a c, \text { in }}-p_{a c, \text { out }}\right)} \\
& T_{a c, \text { out }}=T_{a c, \text { in }}-\epsilon_{a c}\left(T_{a c, \text { in }}-T_{c o o l}\right)
\end{aligned}
$$

where the cooler equivalent area, $A_{a c}$, is not a geometrical area but instead it is used as a tuning parameter. The areas are named; $A_{a c, 1}, A_{a c, 2}, A_{a c, e g r}$. The coolant fluid temperature, $T_{\text {cool }}$, assumed constant, and the cooling element effectiveness, $\epsilon_{a c}$, is modeled as a quadratic function of the cooler mass flow as in Theotokatos ${ }^{12}$

$$
\epsilon_{a c}=k_{a c, 1}+k_{a c, 2} W_{a c}+k_{a c, 3} W_{a c}^{2}
$$

where $k_{a c}$ are tuning parameters and the coolant fluid temperature.

The WMC is assumed to remove all condensed water in the flow. The WMC input mass fraction is converted to molar fraction, $\tilde{X}_{\mathrm{H}_{2} \mathrm{O} \text {,in }}$, using the transformation given in Appendix A. The water saturation pressure, $p_{\mathrm{H}_{2} \mathrm{O} \text {,sat }}$, is calculated at the flow temperature. ${ }^{28}$ The ratio between the saturation pressure and the input pressure corresponds to the maximum volume fraction of vapor in the flow. Since we assume that the gas follows the ideal gas law, the volume fraction is equal to the molar fraction. The outlet molar concentration is limited to the saturation value as

$$
\tilde{X}_{\mathrm{H}_{2} \mathrm{O}, \mathrm{out}}=\min \left(\tilde{X}_{\mathrm{H}_{2} \mathrm{O}, \text { in }}, \frac{p_{\mathrm{H}_{2} \mathrm{O}, \text { sat }}\left(T_{a c, \text { out }}\right)}{p_{a c, \text { out }}}\right)
$$

If the water molar concentration is higher than the saturation value, the condensed water is removed and the output mass flow and mass fraction vector are recalculated accordingly.

The restrictions from the ambient to the compressors inlet control volume and the turbines outlet control volume are also modeled as incompressible turbulent restrictions. The tuning parameters are named; $A_{\text {in }}, A_{\text {out }}$. They define the inlet and outlet flows to the engine, see $W_{\text {in }}$ and $W_{\text {out }}$ in Figure 1. Where the outlet temperature of these two restrictions is assumed to be equal to the inlet.

\subsection{Auxiliary Blowers}

The engine auxiliary blowers are responsible for providing sufficient air mass flow at low loads where the turbochargers efficiency is low. As done in Guan et al., ${ }^{13}$ the pressure increase is modeled as a quadratic function of the blower volume flow. This relation is then inverted ${ }^{21}$ to have the pressure difference as input and the volume flow as an output of the model.

$$
\begin{aligned}
& W_{A u x}=\frac{p_{\text {mix }}\left(k_{A u x, 1}+k_{A u x, 2} \sqrt{k_{A u x, 3}-\left(p_{\text {scav }}-p_{\text {mix }}\right)}\right)}{R_{\text {mix }} T_{\text {mix }}} \\
& T_{\text {Aux }, \text { out }}=T_{\text {mix }}+\frac{T_{\text {mix }}}{\eta_{A u x}}\left\{\left(\frac{p_{\text {scav }}}{p_{\text {mix }}}\right)^{\frac{\gamma_{\text {mix }}-1}{\gamma_{\text {mix }}}}-1\right\}
\end{aligned}
$$

Unfortunately, there is little information about the performance of the auxiliary blower. Only two operating points of volume flow and pressure rise are specified at 
the operating blower speed, and are used to adjust the model parameters $k_{A u x, 1}, k_{A u x, 2}$ and $k_{A u x, 3}$. The auxiliary blower output temperature (13b) is modeled using the isentropic efficiency definition and assuming a constant efficiency value.

The auxiliary blowers dynamic behavior during start and stop is also unknown. The time it takes to accelerate the blowers is modeled as a first order system, where the time constant is adjusted to match the measured pressure response. This will be further discussed in Section 5. The time it takes to stop the blowers is also taken as a first order system, with a distinct time constant since decelerating is faster than accelerating. The dynamics can be written as

$$
\frac{d}{d t} u_{A u x, d}= \begin{cases}\frac{u_{A u x}-u_{A u x, d}}{\tau_{A u x, s t a r t}}, & \text { for } u_{A u x}-u_{A u x, d}>0 \\ \frac{u_{A u x}-u_{A u x, d}}{\tau_{A u x, s t o p}}, & \text { for } u_{A u x}-u_{A u x, d}<0\end{cases}
$$

where the value of $u_{A u x, d}$, follows dynamically the ordered start or stop step signal $u_{A u x}$. The output of (14) is used to scale the flow of the blower during start and stop as

$$
W_{\text {Aux }, d}=u_{A u x, d} W_{\text {Aux }}
$$

Note that when $u_{A u x, d}$ has reached $u_{A u x}$ during a start, its value is 1 and thus $W_{A u x, d}=W_{A u x}$. Hence, the mass flow scaling is only active when the control signal $u_{A u x}$ changes.

The one-way valve between the Gas Mixer and Scavenging control volumes is modeled as an incompressible restriction (10a), without temperature change, using (9b), and with tuning parameter $A_{A u x}$. This restriction starts to govern the flow as soon as the pressure in the scavenging manifold is lower than the pressure in the gas mixer. Which is the normal case when the Auxiliary blowers are not enabled since no backflow is allowed through the blowers.

\subsection{EGR Blowers}

The mass flow provided by the EGR blowers is modeled using the non-dimensional parameters $\Psi$ and $\Phi$, which are assumed to follow an elliptical relation. This model has been used previously for EGR blowers. ${ }^{20-22}$ The use of the non-dimensional parameters is also motivated since they operate at varying speeds but there are only performance measurements for a single speed line. Thus, the non-dimensional parameters give a convenient way to extrapolate the blower performance at different blower speeds, as it is shown in Eriksson ${ }^{29}$ for automotive compressors. The structure of the model is as follows

$$
\begin{aligned}
& W_{e b}=\frac{p_{e b, i n} D_{e b}^{3} \Phi \omega_{e b}}{2 R_{e b, i n} T_{e b, i n}} \\
& T_{e b, \text { out }}=T_{e b, i n}
\end{aligned}
$$

where $D_{e b}$ is the diameter of the blower impeller, and $\Phi$ is computed from the elliptical relation with $\Psi$ and the current operating conditions, as described in ${ }^{20,22}$. The blower temperature at the outlet is assumed to be equal to the inlet since the temperature measurements indicate a very low temperature rise when the blowers operate.

\subsection{Cylinders}

To improve the cylinder model compared to the previous publications ${ }^{20,21}$ was one of the primary objectives of this work. The main interest is that the model resembles as much as possible the physical response of the real engine to changes in control inputs. The cylinder pressure control inputs are; the opening and closing of the exhaust valve (EVO and EVC), the fuel injection angle $\alpha_{i n j}$, and the fuel index $Y$ that defines the injected fuel mass flow $W_{f}$.

One option would be to implement a crank resolved cylinder pressure model as in Guan et al. ${ }^{14}$ However, this will conflict with the objective to have a faster than real time MVEM. In order to improve the cylinder model and still keep it fast, an analytic cylinder pressure model is developed by adapting the four-stroke model described in Eriksson and Andersson ${ }^{30}$ to a two-stroke marine diesel engine. The six cylinders are assumed to operate identically so that one cycle computation will represent all six.

A diagram of the cylinder pressure model is shown in Figure 2, where the different key points are numbered in a similar way as in the ideal Otto cycle. ${ }^{6}$ Note that the start of compression point, point 1 , depends on the control input EVC. The point 3 corresponds to the end of combustion. The end of expansion and start of the blowdown process, point 4 , is controlled by EVO. Point 5 is the end of the blowdown process, which is defined by the fixed angle when the intake ports open, IPO. All thermodynamic properties, e.g., $c_{p}, c_{v}, R$ and $\gamma$, are calculated at the different parts of the cycle as a function of the gas composition and temperature with the help of the NASA polynomials. ${ }^{25}$ Appendix A contains more information about these calculations.

3.7.1 Cylinder Scavenging Scavenging is the process of removing the burned gases from the cylinder and fill it with unburned gas from the scavenging manifold. It is a complicated process involving many physical interactions like heat transfer and fluid dynamics, and it requires advanced models if high accuracy has to be achieved. The goal here is to capture the main characteristics of the scavenging process while keeping a low complexity and fast analytical model.

CFD analysis of similar uniflow scavenged engines show that the scavenging efficiency is usually very high, and the process involves a displacement and mixing of gases, see, i.e., ${ }^{31-33}$. Based on CFD simulations, a simple analytic model for the scavenging efficiency is defined in Andersen. ${ }^{31}$ It is based on a modification of the classical perfect displacement model. ${ }^{34}$ The proposed model also 


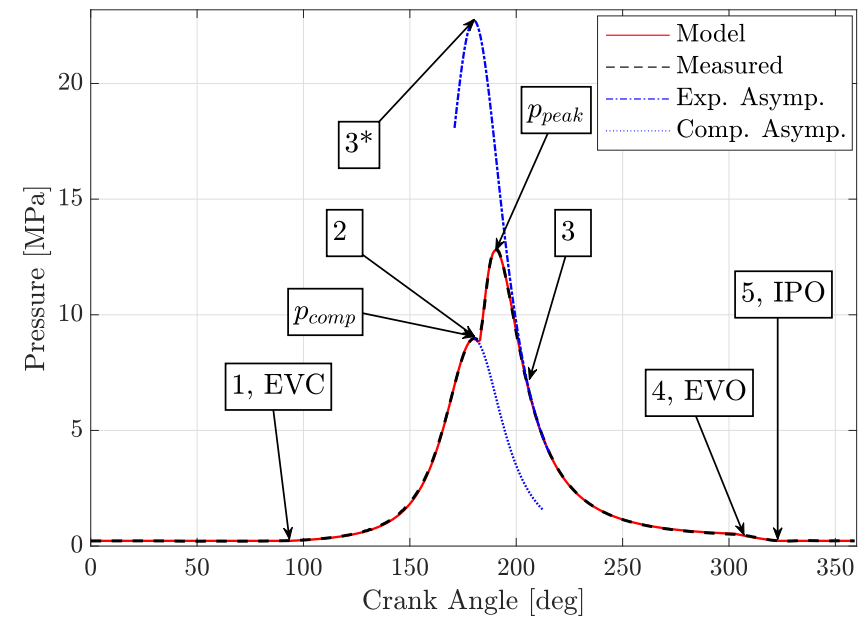

Figure 2. Diagram of the measured cylinder pressure in thick dashed line together with the modeled cylinder pressure in solid line. The compression and expansion asymptotes are depicted in dotted and dashed-dotted lines respectively. The numbers correspond to the different key points of the ideal Otto thermodynamic cycle.

contains an extra submodel to account for the scavenging process that occurs during the push out phase. According to Andersen, ${ }^{31}$ it is important to consider the expelled burned gases when the scavenging ports are closed but the piston moves upwards, and the exhaust valve is still open. The model equation with the volumetric and push out terms is written as

$\eta_{\text {scav }}=\underbrace{a\left[1+\frac{\rho_{\text {exh }}}{\rho_{\text {scav }}}\left(\frac{1}{D R}-1\right)\right]+b}_{d \psi_{\text {vol }}}+\underbrace{c}_{d \psi_{\text {push }, \text { out }}}$

where $D R$ corresponds to the delivery ratio. ${ }^{31,34}$ The parameters $a$ and $b$ are taken as in Andersen. ${ }^{31}$ Since the cylinder volumes are different than the original ones, ${ }^{31}$ the constants $c$ and $d$ are scaled to have similar output values for the push out term, $d \psi_{\text {push,out }}$, as the values reported in Andersen. ${ }^{31}$

3.7.2 Cylinder Mass Flow The mass flow going through the cylinders is modeled as is commonly done for MVEM of two-stroke engines. ${ }^{8,12,20}$ A compressible restriction defined as (9a) is used with a fixed equivalent area as a tuning parameter, $A_{c y l}$. The flow through the cylinders is named delivered flow, $W_{\text {del }}$.

The fuel mass flow is a function of the ordered fuel index, $Y$, and the current engine speed

$$
W_{f}=k_{f, 1}+k_{f, 2} Y \omega_{e n g}
$$

where the parameters $k_{f, 1}$ and $k_{f, 2}$ are adjusted with shop test fuel mass flow measurements and kept constant. The mass flow exiting the cylinders is

$$
W_{c y l}=W_{d e l}+W_{f}
$$

Note that to calculate mass from the mass flows, the engine speed in rps is used.

3.7.3 Compression Pressure The compression pressure asymptote is modeled as a polytropic process starting at the point 1, see Figure 2. Pressure and temperature are calculated as

$$
\begin{aligned}
& p_{c o}(\theta)=p_{1}\left(\frac{V_{1}}{V(\theta)}\right)^{\gamma_{c o}} \\
& T_{c o}(\theta)=p_{1}\left(\frac{V_{1}}{V(\theta)}\right)^{\gamma_{c o}-1}
\end{aligned}
$$

where the polytropic exponent, $\gamma_{c o}$, is calculated with temperature and composition at EVC and kept constant through the compression process.

$$
\gamma_{c o}=\gamma\left(T_{1}, X_{1}\right)
$$

During scavenging and push out, from the Intake Port Opening (IPO) to EVC the cylinder pressure is somewhere between the scavenging and the exhaust pressures. This can be observed on the cylinder pressure measurements, and it is also described in Lamas et al. ${ }^{33}$ However, to keep the model simple, the cylinder pressure is assumed to be equal to the scavenging pressure, $p_{\text {scav }}$ between IPO and EVC. Thus, $p_{1}=p_{\text {scav }}$.

Determining the temperature at EVC is more difficult. First, because no cylinder temperature measurements are available, and because the complex scavenging process has a significant effect on the cylinder temperature during the open phase. The starting temperature is computed as in Eriksson and Andersson, ${ }^{30}$ a mixture of the fresh air at scavenging temperature and the unscavenged gases at the previous cycle final temperature. Once the pressure and the temperature are determined, the trapped mass in the cylinder is calculated using the ideal gas law.

3.7.4 Expansion Pressure A polytropic expansion asymptote is used to model the expansion process, ${ }^{30}$ see Figure 2. The asymptote is defined as

$$
\begin{aligned}
& p_{e x}(\theta)=p_{3^{*}}\left(\frac{V_{2}}{V(\theta)}\right)^{\gamma_{e x}} \\
& T_{e x}(\theta)=T_{3^{*}}\left(\frac{V_{2}}{V(\theta)}\right)^{\gamma_{e x}-1}
\end{aligned}
$$

where the polytropic exponent, $\gamma_{e x}$, is calculated with temperature and composition at the hypothetical point $3^{*}$ and kept constant through the expansion process.

$$
\gamma_{e x}=\gamma\left(T_{3^{*}}, X_{3^{*}}\right)
$$


This hypothetical cycle point $3^{*}$, which defines the expansion asymptote, is determined by adding the combustion temperature increase described in Eriksson and Andersen ${ }^{30}$ to the temperature at point 2.

$$
\begin{aligned}
\Delta T & =\frac{m_{f} q_{L H V} \eta_{f}\left(\alpha_{i n j}\right)}{c_{v}\left(T_{2}, X_{2}\right)\left(m_{f}+m_{\text {trap }}\right)} \\
T_{3^{*}} & =T_{2}+\Delta T
\end{aligned}
$$

With $T_{3^{*}}$ and using the ideal gas law, the pressure can be determined at the cycle point $3^{*}$, see Figure 2 . The burned gas mass fraction at point $3^{*}, X_{3^{*}}$, is computed assuming complete burning of the injected fuel and the original composition of the charge trapped in the cylinder, as described in Nielsen et al. ${ }^{22}$ The efficiency term in (26), $\eta_{f}$, is used to account for pressure and temperature variations in the expansion part of the cycle depending on the injection angle, $\alpha_{i n j}$. It will be further discussed in the following Section.

3.7.5 Combustion Interpolation The combustion part of the cycle is modeled as an interpolation between the two asymptotes, see Figure 2 for a graphical example. The interpolation is done with the help of a Vibe function. ${ }^{30}$

$$
\begin{aligned}
& P R(\theta)=1-e^{-a\left(\frac{\theta-\theta_{o}}{\Delta \theta}\right)^{m+1}} \\
& p_{c y l}(\theta)=(1-P R(\theta)) p_{c o}(\theta)+P R(\theta) p_{e x}(\theta)
\end{aligned}
$$

The Vibe function has four parameters that need to be estimated; $a, m, \theta_{o}$ and $\Delta \theta$. Note that the Vibe function is over-parameterized, ${ }^{3}$ since different combinations of parameters can give the same output shape. To avoid this, the parameter $a$ value is fixed to 5.8. Separated least squares problems are solved for each measured cylinder pressure to obtain the remaining three Vibe parameters and the efficiency term, $\eta_{f}$, for each operating point. Then the relations between the obtained Vibe parameters and other model signals and inputs are investigated to find suitable models that define an overall accurate combustion interpolation model.

The parameter $\theta_{o}$ in (28) defines the crank angle where the cylinder pressure starts to rise from the compression asymptote. Hence, the difference between the estimated value of $\theta_{o}$ and the injection angle is assumed to be the ignition delay

$$
\theta_{\text {delay }}=\theta_{o}-\alpha_{i n j}
$$

The ignition delay is modeled using an Arrhenius type formula as described in Chapter 10 of Heywood. ${ }^{6}$ Usually, the pre-exponential term, $C_{A}$, contains a pressure dependence term. ${ }^{35}$ However here it is kept constant because with two parameters the agreement to the calculated data is similar as if the pressure dependence term is included. The delay is written as

$$
\theta_{\text {delay }}=\frac{180 \omega_{\text {eng }}}{\pi}\left(C_{A} e^{\frac{E_{A}}{T}}\right)
$$
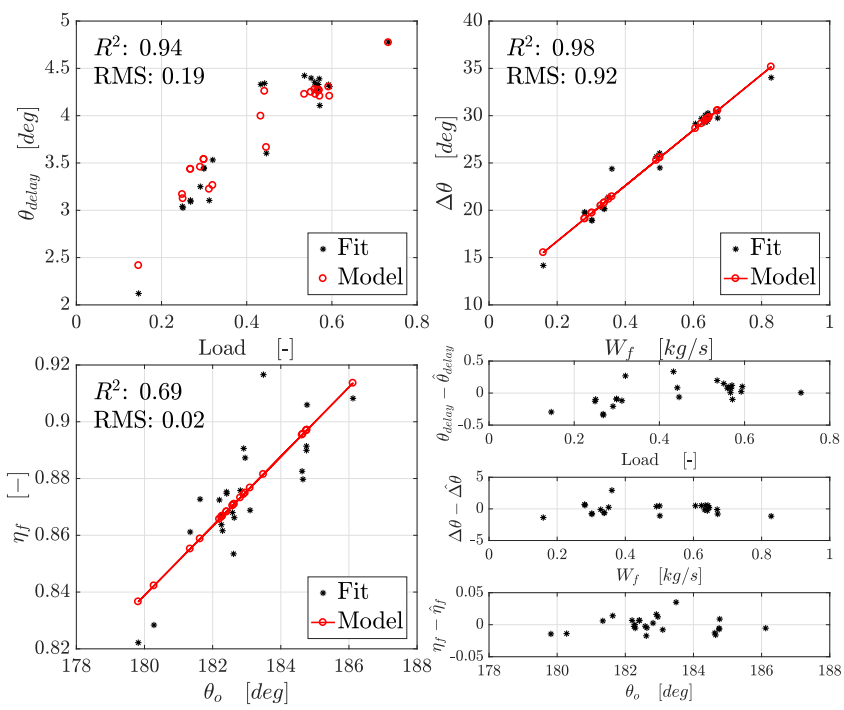

Figure 3. Ignition delay model in the top left, combustion duration model in the top right and efficiency model in the bottom left. Stars correspond to the separated fit for each pressure measurement. Circles represent the proposed model. Each model fit contains the root-mean-square error (RMS) and the R-squared value. The errors of each of the three models are plotted in the bottom right plots.

where $C_{A}$, and the activation term, $E_{A}$, are adjusted to the calculated ignition delay values. The engine speed, $\omega_{\text {eng }}$, is used to convert time to crank angle and $T$ is the gas temperature when the fuel is injected. The model fit with only two parameters is reasonable as can be seen in Figure 3.

The combustion duration, represented by $\Delta \theta$ is modeled as a linear function of the injected fuel mass flow.

$$
\Delta \theta=k_{\Delta \theta, 1}+k_{\Delta \theta, 2} W_{f}
$$

This gives a good representation of the estimated values as can be seen in Figure 3.

Defining the efficiency factor, $\eta_{f}$, as function of injection angle is motivated by Figure 7.28 in Eriksson and Nielsen. ${ }^{3}$ Earlier injection results in higher peak pressures and temperatures which consequently lead to more heat losses. On the other hand, later injection reduces the peak pressures but leads to higher expansion pressures and temperatures later in the cycle. Thus, to capture this, the efficiency is taken as a linear function of the start of combustion, $\theta_{o}$.

$$
\eta_{f}=k_{\eta_{f}, 1}+k_{\eta_{f}, 2} \theta_{o}
$$

As can be seen in Figure 3 a linear model captures reasonably well the estimated values from the separated least squares problems.

Finally, the parameter $m$ is taken as a constant and initially set as the mean value of the estimated values 
to keep the model complexity low and avoid overparameterization. In total, there are seven parameters to be determined for the cylinder pressure model.

3.7.6 Blowdown The blowdown phase starts at the EVO and finishes when the scavenging process begins at IPO. During the blowdown, the measured pressure decreases fast to the lower pressure at IPO, and this decrease can be approximated quite well with a linear model. Since we already defined the cylinder pressure at IPO being equal to $p_{\text {scav }}$, the line connecting the two pressure points is entirely defined, and it can be seen in Figure 2.

The temperature at EVO can be computed using the pressure asymptote, the trapped mass in the cylinder and the ideal gas law. However, the temperature at IPO requires some more assumptions if one wants to keep the model analytic and simple. When the exhaust valve is open, an energy balance is applied to the open system. Following the reasoning in Section 5.5 of Çengel and Boles, ${ }^{28}$ a uniform-flow process can represent reasonably well the real undergoing process. The primary assumption is that the enthalpy of the exiting gas is considered constant, and it is computed as the enthalpy of the gas at EVO, i.e., a throttling process. This simplifies the model since an explicit equation for $T_{5}$ can be derived. The energy balance can be written as

$$
m_{5} c_{v} T_{5}-m_{4} c_{v} T_{4}=-\Omega_{4,5}-\left(m_{4}-m_{5}\right) c_{p} T_{4}
$$

where $\Omega$ is the work done by the gas to the piston from EVO to IPO. Since a linear pressure decrease from EVO to IPO has been assumed, the work can be computed as

$$
\Omega_{4,5}=\frac{\left(p_{4}-p_{5}\right)\left(V_{5}-V_{4}\right)}{2}
$$

Using the ideal gas law to compute the mass inside the cylinder at IPO the explicit expression for $T_{5}$ can be written as

$$
T_{5}=\frac{\frac{p_{5} V_{5} c_{p} T_{4}}{R}}{m_{4} T_{4}\left(c_{p}-c_{v}\right)+\Omega_{4,5}+\frac{c_{v} p_{5} V_{5}}{R}}
$$

where the dependencies of temperature and composition of $c_{v}, c_{p}$ and $R$ have been dropped only in the notation. The thermodynamic properties have been computed with the temperature and concentration at EVO to be able to keep (36) explicit.

3.7.7 Cylinder Outlet Temperature Model Since different mass flows at different temperatures enter the exhaust manifold during the cycle, a final balance is made to compute the resulting cylinder out temperature. This balance is done assuming a perfect mixing process at constant pressure. Thus, the final expression is defined as

$$
T_{c y l}=\frac{\left(m_{4}-m_{5}\right) c_{p} T_{4}+m_{s h} c_{p} T_{s c a v}+\eta_{s c a v} m_{5} c_{p} T_{5}}{\left(m_{4}-m_{5}\right) c_{p}+m_{s h} c_{p}+\eta_{s c a v} m_{5} c_{p}}
$$

where $m_{s h}$ corresponds to the equivalent short-circuited mass during one two-stroke cycle. The short-circuited mass is computed as the delivered mass minus the trapped mass plus the part of the cylinder mass that is not scavenged and remains in the cylinder. This is written as

$$
m_{s h}=m_{\text {del }}-m_{\text {trap }}+\left(1-\eta_{\text {scav }}\right) m_{5}
$$

According to CFD analyses of the scavenging process, ${ }^{32,33}$ there is almost no short-circuit of pure scavenge air during the scavenging phase, and the mass that is not trapped in the cylinder corresponds to a mix of fresh and burned gases. Nevertheless, to keep the model simple, it is assumed here that the non-trapped gases are expelled at a temperature equal to $T_{\text {scav }}$.

3.7.8 Cylinder Outlet Gas Composition The mass fraction after the combustion is calculated assuming complete combustion of the injected fuel. Without including the nitrogen explicitly and considering lean combustion, the chemical reaction can be written as ${ }^{36}$

$$
\mathrm{CH}_{y} \mathrm{~S}_{z}+\left(1+\frac{y}{4}+z\right) \mathrm{O}_{2} \rightarrow \mathrm{CO}_{2}+\frac{y}{2} \mathrm{H}_{2} \mathrm{O}+z \mathrm{SO}_{2}
$$

where $y$ is the hydrogen to carbon ratio and $z$ is the sulfur to carbon ratio in the fuel, which are known parameters. Knowing the trapped and fuel masses, the mass fraction after the combustion, $X_{c o m b}$, can be calculated in a similar way as in Nielsen et al. ${ }^{22}$ but getting masses instead of moles as output. For the oxygen mass fraction, it is computed as

$$
X_{O_{2}, c o m b}=\frac{m_{\text {trap }} X_{O_{2}, i n}-\left(1+\frac{y}{4}+z\right) \frac{M_{O_{2}}}{M_{f}} m_{f}}{m_{\text {trap }}+m_{f}}
$$

For the rest of the gas concentrations, similar equations are derived based on (39). Finally, the mass fraction exiting the cylinders, $X_{c y l}$ can be computed by mixing the burned gases mass fraction with the short-circuited scavenging mass fraction as follows.

$$
X_{c y l}=\frac{X_{c o m b}\left(m_{t r a p}+m_{f}\right)+X_{s c a v}\left(m_{d e l}-m_{\text {trap }}\right)}{m_{d e l}+m_{f}}
$$

\subsection{Engine Brake Power}

The indicated work, $\Omega_{i}$, is computed as the integral of the pressure over the cylinder volume. The integration is done analytically for the polytropic compression and expansion processes, $\Omega_{1,2}$ and $\Omega_{3,4}$, for the blowdown, see (35), and for the constant pressure between IPO and EVC, $\Omega_{5,1}$. On the other hand, for the combustion phase, $\Omega_{2,3}$, the integral is solved numerically. Since all cylinders are considered to work under the same conditions, the indicated power is computed using the current engine speed and multiplying 
the produced power by the number of engine cylinders, $n_{c y l}$.

$$
P_{i}=\frac{\omega_{\text {eng }} n_{c y l}\left(\Omega_{1,2}+\Omega_{2,3}+\Omega_{3,4}+\Omega_{4,5}+\Omega_{5,1}\right)}{2 \pi}
$$

The friction mean effective pressure, $p_{m e f}$, represents the friction power losses normalized to the engine displaced volume. These friction losses are modeled as a linear function of engine speed

$$
p_{m e f}=k_{f r, 1}+k_{f r, 2} \omega_{e n g}
$$

where parameters $k_{f r, 1}$ and $k_{f r, 2}$ are tuning parameters. Finally, the engine brake power, which is the effective power transferred to the propeller shaft, is calculated as

$$
P_{\text {brake }}=P_{i}-\frac{V_{d} n_{c y l} \omega_{e n g}}{2 \pi} p_{m e f}
$$

where $V_{d}$ is the displaced volume of one cylinder.

\subsection{Cylinder Pressure Model vs Measurements}

Figure 4 contains several pressure diagrams for different relative loads and engine running modes to show that the measured pressure can be predicted with the proposed model. The mean absolute relative errors for the indicated power, the peak cylinder pressure and the cylinder compression pressure at point 2 are; $1.49 \%, 1.48 \%$ and $0.36 \%$ respectively.

\subsection{Exhaust Manifold Temperature Model}

Having an accurate exhaust temperature model is important for the overall engine model accuracy. This is because the exhaust temperature together with the pressure sets the available power to the turbines, which in turn determines the rest of the model pressures. A heat transfer model is implemented to account for the gas temperature loss from the cylinder outlet to the turbine inlets.

Initially, a model consisting of the heat transfer from the gas temperature to the ambient was considered. In stationary conditions, this model manages to get accurate predictions of the measured temperatures. However, during transients, the temperature dynamics are very quick and reactive to changes in mass flow through the cylinders and to injected fuel. This makes the whole engine model dynamics too fast compared to the measured signals. To slow down the model dynamics, the thermal inertia is increased by considering the wall temperature of the exhaust gas receiver as a model state. This slows down the exhaust temperature response since the thermal inertia of the wall is large.

The implemented heat transfer model is described as Model 3 in Eriksson. ${ }^{37}$ Where the dynamic wall
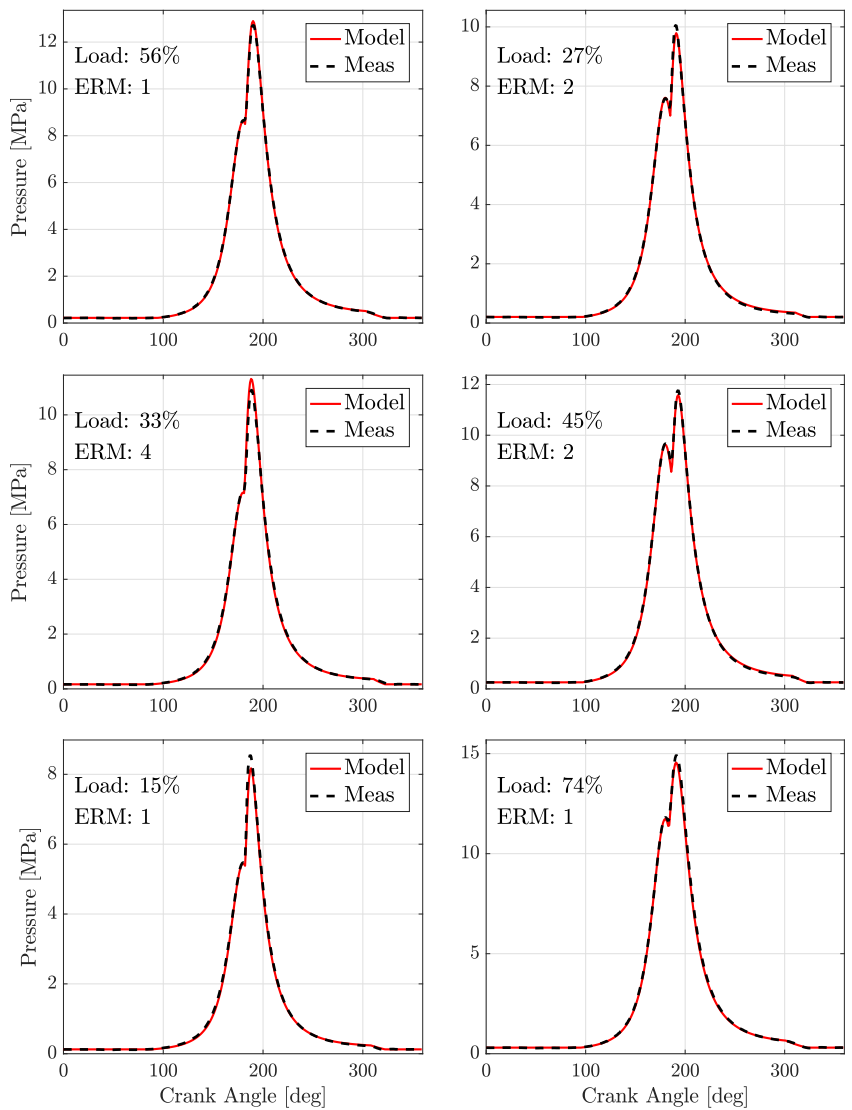

Figure 4. Modeled cylinder pressure in solid lines compared to the measured in dashed lines for different percentages of the maximum engine load and the corresponding engine running mode (ERM)

temperature becomes a model state. The dynamic equation for the wall temperature is defined as

$$
\frac{d T_{e w}}{d t} m_{e w} c_{e w}=\dot{Q}_{i}-\dot{Q}_{e}
$$

where $\dot{Q}_{i}$ is the internal heat transfer from the exhaust gas to the manifold wall, calculated as

$$
\dot{Q}_{i}=W_{c y l} c_{p}\left(T_{c y l}-T_{e w}\right)\left(1-e^{-\frac{h_{c v, i} A_{e w}}{W_{c y l} c_{p}}}\right)
$$

where the convection coefficient, $h_{c v, i}$, is considered as a tuning parameter, and the exhaust wall area, $A_{e w}$ is fixed to its geometrical value. The heat transfer calculated in (46), is inserted in (4c) to get the exhaust manifold temperature. On the other hand, $\dot{Q}_{e}$ is the external heat transfer from the exhaust manifold wall to the surroundings

$\dot{Q}_{e}=F_{v} \epsilon \sigma A_{e w}\left(T_{e w}^{4}-T_{a m b}^{4}\right)+h_{c v, e} A_{e w}\left(T_{e w}-T_{a m b}\right)$

The product of the gray body view factor and the emissivity, $F_{v} \cdot \epsilon$, is considered a tuning parameter. The 
convection coefficient, $h_{c v, e}$, is also a tuning parameter. The original model $3^{37}$ considers conduction to the engine block. However, since the engine block temperature is not measured, in the implementation this temperature is taken as the ambient temperature. In practice, this assumption approximates the conduction heat transfer lumped into the convection, since the coefficient is estimated to fit the engine measurements.

\subsection{Oxygen Sensor}

Three different oxygen sensors measure the molar oxygen fraction of the gas in the scavenging manifold. A complex scavenging gas extraction system is installed in the engine, with the purpose to have a favorable pressure and temperature for the oxygen sensors. This gas extraction system introduces uncertainty in the dynamic response of the oxygen sensor setup. The oxygen sensor response can be approximated with a first order system with a pure delay $^{22}$

$$
\frac{d}{d t} \tilde{O}_{2, s e n}(t)=\frac{\tilde{O}_{2, s c a v}\left(t-\Delta t_{O s}\right)-\tilde{O}_{2, s e n}(t)}{\tau_{O s}}
$$

where $\tilde{O}_{2, \text { scav }}$ is the scavenging molar fraction and $\tilde{O}_{2, \text { sen }}$ is the sensor reading. The time constants, $\tau_{O s}$ and $\Delta t_{O s}$, are both expected to be in the range of $10-20 \mathrm{~s}$ depending on the scavenging pressure and gas extracting clogging conditions.

\subsection{Exhaust Temperature Sensor}

The dynamic response of the exhaust temperature sensor is modeled as a first order system to compare the sensor signal to the modeled exhaust temperature.

$$
\frac{d}{d t} T_{e x h, s e n}(t)=\frac{T_{e x h}(t)-T_{e x h, s e n}(t)}{\tau_{T s}}
$$

$T_{\text {exh,sen }}$ is the exhaust temperature with the sensor dynamics, and $T_{e x h}$ is the exhaust temperature state of the exhaust manifold, see Figure 1. The time constant $\tau_{T s}$ is unknown, but a value of $30 \mathrm{~s}$ gives a response similar to the measured temperature. Section 5.2 contains further discussion about the agreement of the modeled and measured exhaust temperature signals.

\section{Model Parameterization}

The proposed MVEM has three main internal feedback systems, the EGR loop, and the two turbochargers. Hence, the modeling errors of any subsystem will be coupled to the rest of the model and amplified. For instance, the errors in the exhaust temperature will affect the turbine power production which in turn will change the scavenging pressure, and this finally will modify the exhaust temperature prediction again. Due to this, balancing out the complete model, by readjusting the model parameters is very important to obtain an overall accurate model.

In the following sections, the relative error is defined as

$$
e[k]=\frac{y_{\text {mod }}[k]-y_{\text {meas }}[k]}{1 / N \sum_{j=1}^{N} y_{\text {meas }}[j]}
$$

where $y_{\text {mod }}$ is the model signal and $y_{\text {meas }}$ corresponds to the measured signal. Before computing the mean value, the absolute value of the relative error is taken to avoid canceling out errors of different sign. Moreover, the relative errors in the text and tables are given in percentage, multiplying (50) by 100 .

\subsection{Initialization}

First of all, the parameters for the turbomachinery models are estimated and kept fixed. The turbocharger models are parameterized using the provided SAE maps. The compressor model is parameterized with the algorithm described in Llamas and Eriksson. ${ }^{26}$ The EGR and auxiliary blowers are also parameterized using the performance data.

During the parameterization of automotive MVEMs, the measured mass flow is an advantageous signal to be able to parameterize the models of the different engine components by solving separated least squares problems. Unfortunately, this procedure cannot be followed here since the engine mass flow is not measured, which complicates the initialization of the model parameters. A way to remedy this issue is to use the compressor model together with the measured speed and differential pressure to get an estimate of the engine mass flow when EGR is not running. This estimated mass flow is used to initialize the parameters of the valves, the flow restrictions and the coolers before the complete parameterization procedure.

The cylinder pressure model is initialized in Section 3.7 to match the indicated power and cylinder pressure. In total seven parameters corresponding to (28), (31), (32) and (33). However, the cylinder model needs to be adjusted so that the cylinder output temperature agrees with the measurements. Unfortunately, the cylinder outlet temperature is not measured. Hence, the cylinder model is initialized together with the exhaust heat transfer model to match the measured engine brake power and exhaust temperature. The initialization is started with the previously mentioned seven parameters together with five more corresponding to (46), (47) and (43). In order to prevent the parameterization algorithm from moving away from the designed cylinder pressure model described in Section 3.7, two additional signals are used in the objective function since the cylinder pressure measurements are not available 
for the stationary points. These signals are the maximum pressure in the cylinder as well as the compression pressure in the cylinder. These pressures are measured by the engine control system and used to control the combustion process.

\subsection{Stationary Parameterization}

The procedure followed here to solve the parameterization problem is very similar to ones used in previous work. ${ }^{21}$ For each measured stationary point, the engine model is simulated in Simulink with the constant measured input signals. Once the model reaches stationarity, the model output values are used to compute the relative errors with the measured signals, using (50). This method involves a large number of dynamic simulations per solver iteration which can make the complete process very computationally demanding. However, for a given solver iteration, the required dynamic simulations are independent of each other so the process can be parallelized using the Matlab Parallel Computing Toolbox.

A least squares problem is then formed by stacking the relative errors for each considered stationary point and measured signal. The used solver is the Matlab Optimization Toolbox lsqnonlin, based on GaussNewton method with numerical approximation of the jacobian.

In the general case the cost function to be minimized is written as

$$
V_{\text {stat }}(\varphi)=\frac{1}{N S} \sum_{i=1}^{S} \sum_{n=1}^{N}\left(e_{\text {rel }}^{i}[n]\right)^{2}
$$

where $N$ is the number of stationary points and $S$ is the number of different signals involved in the estimation. The estimated parameters are contained in a vector named $\varphi$. The parameters are subject to bound constraints to keep the solver from choosing unreasonable values.

The complete parameterization is done in steps to ensure that the solver is capable to solve the problem while obtaining reasonable parameters. The steps start with the engine running mode that involves the lowest number of parameters and increase in model complexity, the following subsections contain such steps and how they are performed. Iterating steps 3, 4 and 5 once, updating the current initial parameters with the previous step value has helped to fine tune the model. Finally it is worth mentioning that the optimization problem is highly nonlinear and non-convex, so there is no guarantee that the found solution is the global optimum. In order to ensure that the parameters are a good suitable solution, the proposed steps have been started with different initial values and the obtained parameters have been compared and the best ones are kept. The parameter selection criterion is to check which set of parameters gives the lowest sum of squared residuals.
Step 1 ERM 2 stationary points. Only the main turbocharger is working and there is no EGR operation. The relative errors used in (51) are computed with the measured signals: $p_{\text {scav }}, p_{c, 1}, \omega_{t c, 1}, P_{\text {brake }}$, and $T_{e x h}$. The estimated parameters are

$$
\varphi=\left[A_{a c, 1}, A_{\text {Aux }}, A_{E G B}, A_{c y l}, A_{\text {out }}, A_{\text {in }}, \eta_{\text {mech }, 1}\right]
$$

Step 2 Stationary points with ERM 1 and 2. Both turbochargers are working, but there is no EGR operation. The parameters estimated in the previous step are used as starting point. The relative errors used in (51) are computed with the measured signals: $p_{s c a v}, p_{c, 1}, \omega_{t c, 1}, \omega_{t c, 2}, P_{\text {brake }}$ and $T_{e x h}$. The estimated parameters are

$$
\begin{gathered}
\varphi=\left[A_{a c, 1}, A_{a c, 2}, A_{A u x}, A_{E G B}, A_{c y l},\right. \\
\left.A_{\text {out }}, A_{\text {in }}, \eta_{\text {mech }, 1}, \eta_{\text {mech }, 2}\right]
\end{gathered}
$$

Step 3 Stationary points with ERM 3 and 4, EGR is now running. While keeping the rest of the parameters fixed, the EGR loop flow restrictions are estimated. The relative errors used in (51) are computed with the measured signals: $p_{\text {scav }}$, $p_{c, 1}, \omega_{t c, 1}, P_{\text {brake }}, T_{e x h}, \tilde{X}_{O_{2, s c a v}}, p_{e b, \text { in }}$ and $p_{e b, o u t}$. The estimated parameters are

$$
\varphi=\left[A_{S D V}, A_{a c, e g r}, A_{C O V}\right]
$$

Step 4 Using all stationary points the exhaust manifold heat transfer model is re-parameterized. All other parameters are kept constant. The relative errors used in (51) are computed with the measured signals: $p_{s c a v}, p_{c, 1}, \omega_{t c, 1}$, $\omega_{t c, 2}, P_{\text {brake }}, T_{e x h}$ and $\tilde{X}_{O_{2, s c a v}}$. This step involves three heat transfer coefficients of the exhaust manifold temperature model

$$
\varphi=\left[h_{c v, i}, h_{c v, e}, F_{v} \cdot \epsilon\right]
$$

Step 5 Using all stationary points the restriction parameters are re-parameterized starting with the parameter values obtained in the previous steps. The relative errors used in (51) are computed with the measured signals: $p_{\text {scav }}$, $p_{c, 1}, \omega_{t c, 1}, P_{b r a k e}, T_{e x h}, \tilde{X}_{O_{2, s c a v}}, p_{e b, \text { in }}$ and $p_{e b, o u t}$. The estimated parameters are

$$
\begin{gathered}
\varphi=\left[A_{a c, 1}, A_{a c, 2}, A_{A u x}, A_{E G B}, A_{c y l},\right. \\
\left.A_{\text {out }}, A_{\text {in }}, \eta_{\text {mech }, 1}, \eta_{\text {mech }, 2}\right]
\end{gathered}
$$

\subsection{Dynamic Parameterization}

This final parameterization step involves estimating the parameters that define the dynamic behavior of the model, which corresponds to the turbocharger inertias and the control volume sizes.

A least squares problem that minimizes the dynamic deviation of the measured states can be solved to estimate 
the model dynamic parameters. ${ }^{19-21}$ However, solving this optimization can be complex and it requires suitable transient datasets. Instead, this procedure is simplified here by setting the value of the control volumes to reasonable values of the real pipe volume sizes based on the engine design drawings. The turbocharger inertias are set to the nominal values included in the SAE maps. This is a good strategy to define the model dynamic parameters in case only stationary shop test data is available for parameterization.

The model response with the previously mentioned dynamic parameters is quite good. However, when EGR is started or stopped the model response is faster than the measured. This issue is solved by increasing the main turbocharger inertia by $30 \%$ of the nominal SAE value, which improves the dynamic response of the model. This behavior was also noticed in previous versions of the model, ${ }^{20,21}$ where the exhaust heat transfer was not modeled, and the turbocharger inertia had to be increased substantially more. Sections 5.2 and 6 contain further discussion about this issue.

\section{Model Validation}

The model is validated by comparing the measured signals to the model predictions. First, this is done evaluating the model in stationary conditions. Second, the model is also validated under transient conditions.

\subsection{Stationary Results}

The absolute relative stationary errors are presented in Table 1 for both the parameterization and validation data. The mean relative errors for the tuning data are in general under $3.35 \%$, which is a good indication that the model is capable of predicting the main engine outputs for any load and ERM value. It is worth to mention that the highest errors are when EGR is active, i.e., in ERM 3 and 4. This is expected since the engine becomes more complex when the EGR system is active, more engine components are working, and thus more uncertainty is added to the system. Note also that Table 1 contains the number of stationary points available for each ERM, and the modes 2 and 3 have fewer points than modes 1 and 4 to tune and to compute the errors of the model. Only points with EGR active have been used to compute the molar oxygen concentration error, as well as only points operating at ERM number 1 are used in the second turbocharger speed error calculation.

The Figure 5 depicts the model predictions vs. the measured values for the parameterization data. The overall accuracy of the model is good, from $10 \%$ to almost $90 \%$ loads, with and without EGR operation.

In order to show that the model can achieve accurate predictions not only with the points used in the
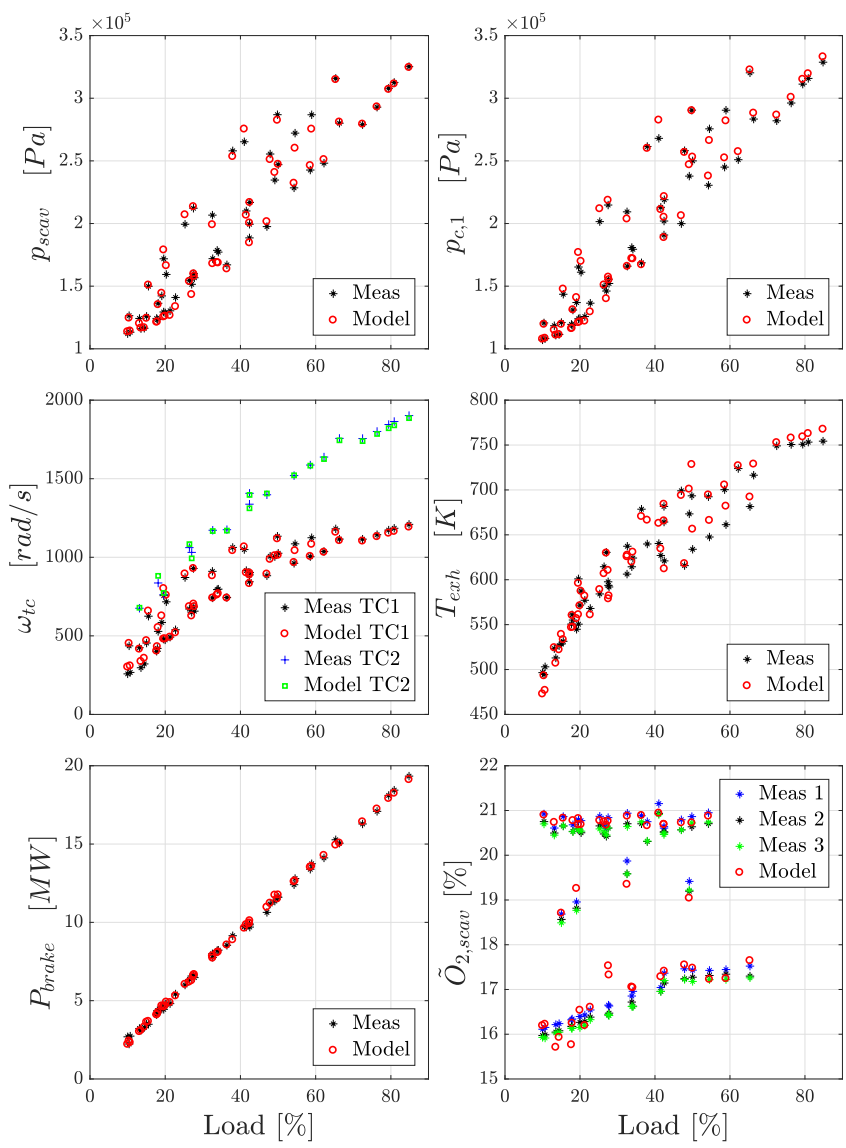

Figure 5. Stationary model predictions vs. measurements for different engine signals using the parameterization stationary data. Note that the molar oxygen concentration has three different sensors in the same location.

parameterization, the model predictions are compared for a set of stationary points not used in the parameterization. These stationary points are named validation points, and their numerical errors are also given in Table 1, and shown graphically in Figure 6. This set of data points are close to the previous parameterization data since they are also extracted from normal operation of the engine during sailing. However, they are not the same operating points. Note that the errors for the ERM 3 are unfortunately computed with a single measured point. This is of course not desirable, but the engine is very seldom operating in this conditions. The errors increase only slightly when going from tuning data to validation data, as seen in Table 1, which is a good indication that the model will be able to predict the engine operation accurately. Table 1 also contains the root-mean-square errors (RMS) for each of the listed measured signals, which indicates the spread of the model errors. The RMS values for estimation and validation data are similar, which is also an indication of good model accuracy. 
Table 1. Absolute relative errors [\%] of the model for both estimation and validation data, separated for different ERMs. The table also contains the combined root-mean-square error (RMS) of all listed residuals.

\begin{tabular}{l||cccc|l||cccc|l}
\multicolumn{1}{l||}{} & \multicolumn{5}{c||}{ Estimation Stationary Points } & \multicolumn{5}{c}{ Validation Stationary Points } \\
\hline ERM & 1 & 2 & 3 & 4 & RMS & 1 & 2 & 3 & 4 & RMS \\
\hline Points & 19 & 9 & 4 & 20 & 52 & 17 & 9 & 1 & 13 & 40 \\
\hline$p_{\text {scav }}$ & 1.30 & 2.53 & 2.21 & 1.87 & $4.66 \cdot 10^{3} \mathrm{~Pa}$ & 1.94 & 2.34 & 3.07 & 1.64 & $4.93 \cdot 10^{3} \mathrm{~Pa}$ \\
$\omega_{t c, 1}$ & 1.35 & 2.99 & 2.88 & 2.95 & $23.26 \mathrm{rad} / \mathrm{s}$ & 1.35 & 2.59 & 0.73 & 2.54 & $20.57 \mathrm{rad} / \mathrm{s}$ \\
$\omega_{t c, 2}$ & 1.14 & - & - & - & $19.04 \mathrm{rad} / \mathrm{s}$ & 1.24 & - & - & - & $21.73 \mathrm{rad} / \mathrm{s}$ \\
$p_{c, 1}$ & 1.98 & 3.16 & 2.56 & 1.78 & $5.30 \cdot 10^{3} \mathrm{~Pa}$ & 2.78 & 3.16 & 4.75 & 1.42 & $5.88 \cdot 10^{3} \mathrm{~Pa}$ \\
$T_{\text {exh }}$ & 0.97 & 2.06 & 2.80 & 1.65 & $12.22 \mathrm{~K}$ & 0.77 & 2.65 & 4.15 & 1.30 & $12.28 \mathrm{~K}$ \\
$P_{\text {brake }}$ & 1.66 & 1.61 & 2.40 & 1.94 & $1.93 \cdot 10^{5} \mathrm{~W}$ & 2.33 & 1.71 & 4.35 & 1.53 & $2.28 \cdot 10^{5} \mathrm{~W}$ \\
$\tilde{X}_{O_{2, \text { scav }}}$ & - & - & 1.54 & 1.69 & $0.34 \%$ & - & - & 1.40 & 2.19 & $0.46 \%$ \\
$p_{\text {eb,in }}$ & - & - & 2.91 & 2.90 & $5.33 \cdot 10^{3} \mathrm{~Pa}$ & - & - & 4.10 & 2.59 & $4.75 \cdot 10^{3} \mathrm{~Pa}$ \\
$p_{\text {eb,out }}$ & - & - & 2.76 & 3.35 & $5.38 \cdot 10^{3} \mathrm{~Pa}$ & - & - & 4.20 & 1.98 & $5.67 \cdot 10^{3} \mathrm{~Pa}$ \\
\hline
\end{tabular}
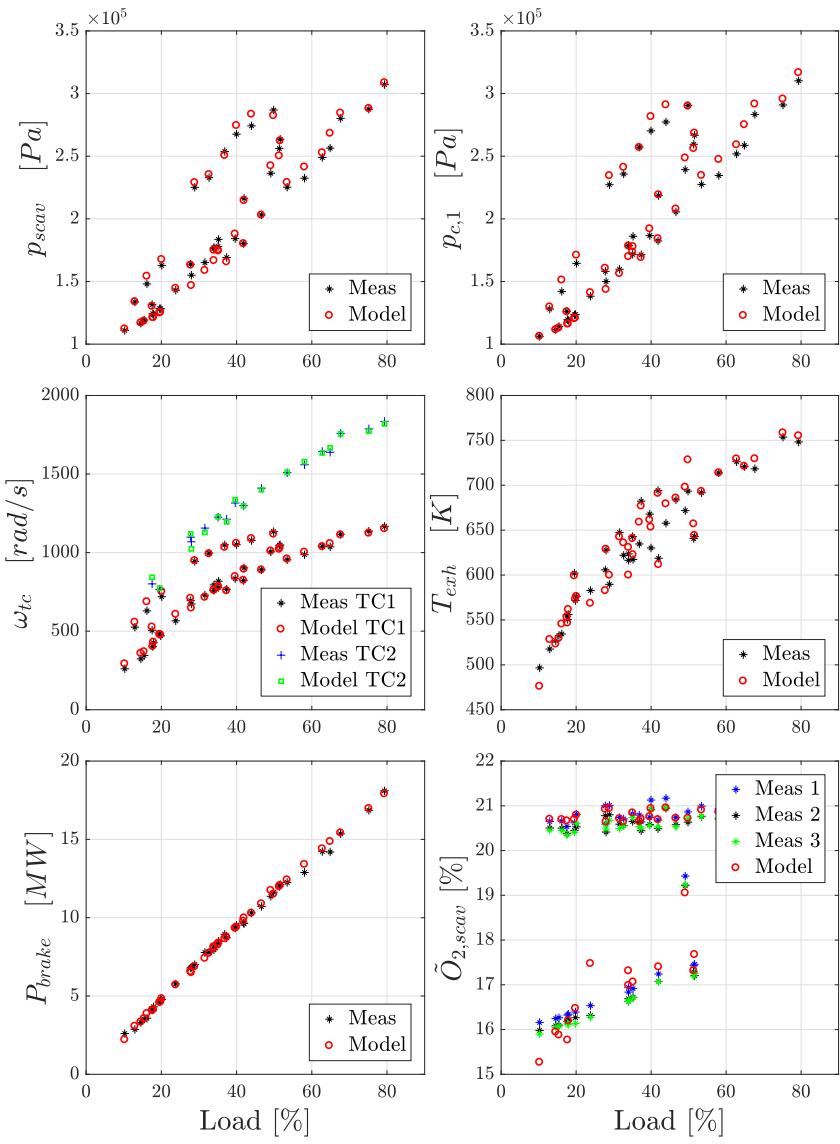

Figure 6. Stationary model predictions vs. measurements for different engine signals using the validation stationary data. Note that the molar oxygen concentration has three different sensors in the same location.

\subsection{Dynamic Results}

Four different dynamic scenarios are simulated and compared to the model predictions to validate the model dynamics. The four scenarios are load increase, load decrease, EGR start and EGR stop. All scenarios are focused on low load operation, which is the most uncertain operating area of the model since the auxiliary blower is running and the turbochargers are operating outside of the mapped area. Moreover, most of the maneuvering transients occur at low loads.

The first scenario with increasing load and the two turbochargers running is depicted in Figure 7. Approximately the engine load goes from $20 \%$ to $35 \%$ with the auxiliary blowers running. The model shows a good agreement with the measured data for both pressure signals. For the turbocharger speed signals the agreement is also good, however, there is a higher stationary error. The fast temperature sensor is plotted together with the exhaust temperature in dashed line and the output of the temperature sensor described in (49). As can be seen, the modeled sensor temperature agrees quite well to the measurement, while the modeled exhaust temperature is faster. A time constant of $30 \mathrm{~s}$ has been found to be a reasonable approximation of the sensor dynamics for all the cases tested. In reality, the sensor might be faster and the real exhaust temperature slower. Unfortunately, this is not possible to determine with certainty with the currently available measurements.

The next scenario, shown in Figure 8, is an engine load decrease. Stationary errors are higher than in the previous case, but the dynamic response of the model compared to pressures and turbocharger speeds is also good. This dynamic dataset has the particularity to start the Auxiliary blower approximately at $450 \mathrm{~s}$. As can be seen in Figure 8, the model is capable of capturing the effect that the auxiliary blower has on the turbocharger speeds and the pressures. The auxiliary blowers start time constant, described in (14), is adjusted to match the dynamic response. This, as discussed in Section 3.5, represents the time it takes for the blowers to accelerate to the working speed.

The third scenario consists of an EGR start and it is depicted in Figure 9. The engine load is constant and close to $20 \%$ of the maximum rated power, the auxiliary 

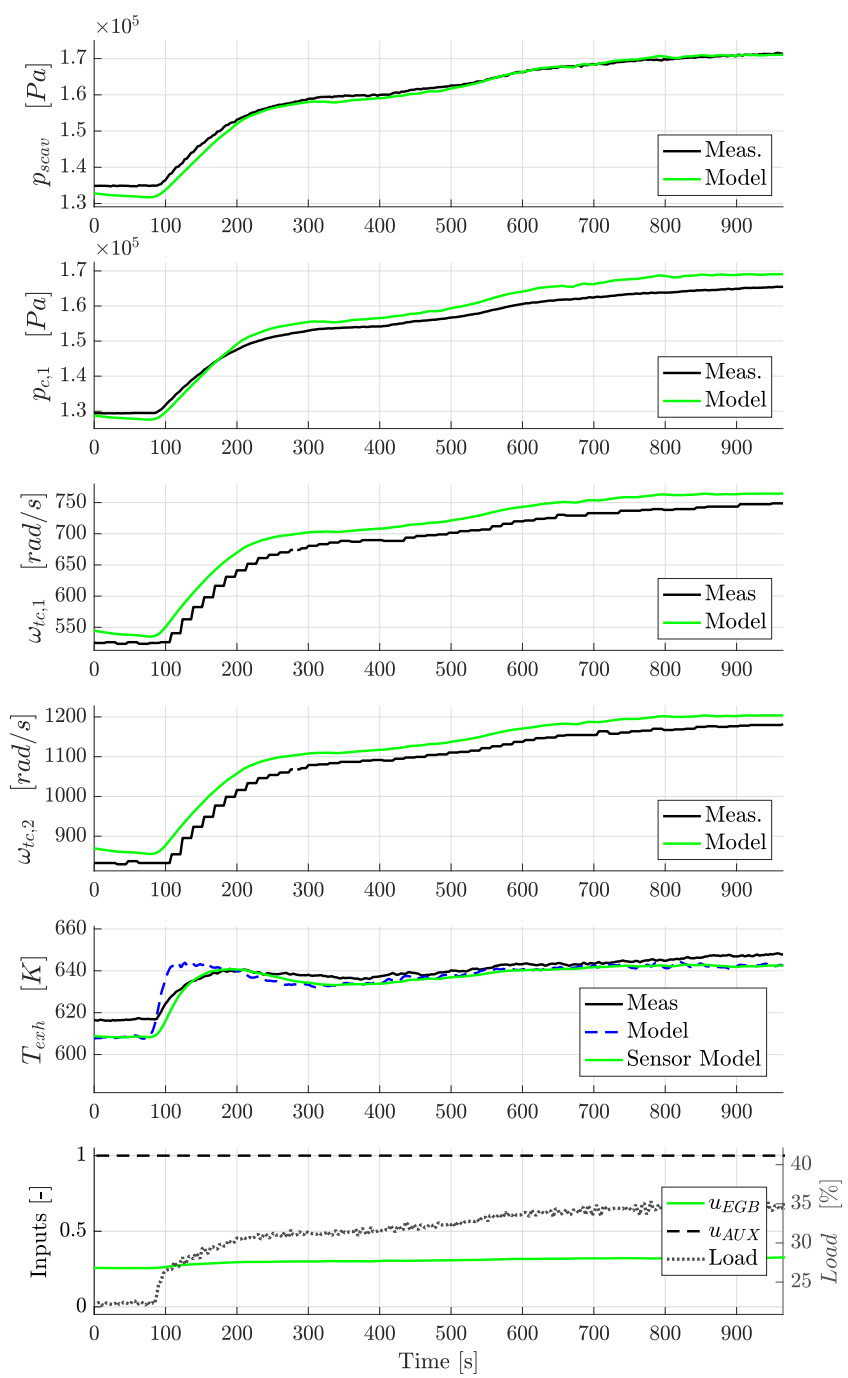

Figure 7. Model transient predictions and measured signals for a Load increase scenario. The right axes in the bottom plot correspond to the percentage of engine load, depicted with a dotted line.

blower is active during the transient. The EGR valve opens approximately at $80 \mathrm{~s}$ and the oxygen level in the manifold starts to drop to later follow the ordered EGR blower speed fluctuation. The agreement between measured and modeled oxygen volume fractions is good. Note that the modeled oxygen is passed through the sensor dynamics model described in (48), to have both signals synchronized. The modeled temperature drops faster than the measurement when EGR starts to flow. However, when the sensor dynamics are included, the temperature response agrees much more to the measured signal.

The last scenario, shown in Figure 10 is an EGR stop at $25 \%$ engine load with the auxiliary blower enabled. The pressure and turbocharger speed dynamics are good, despite
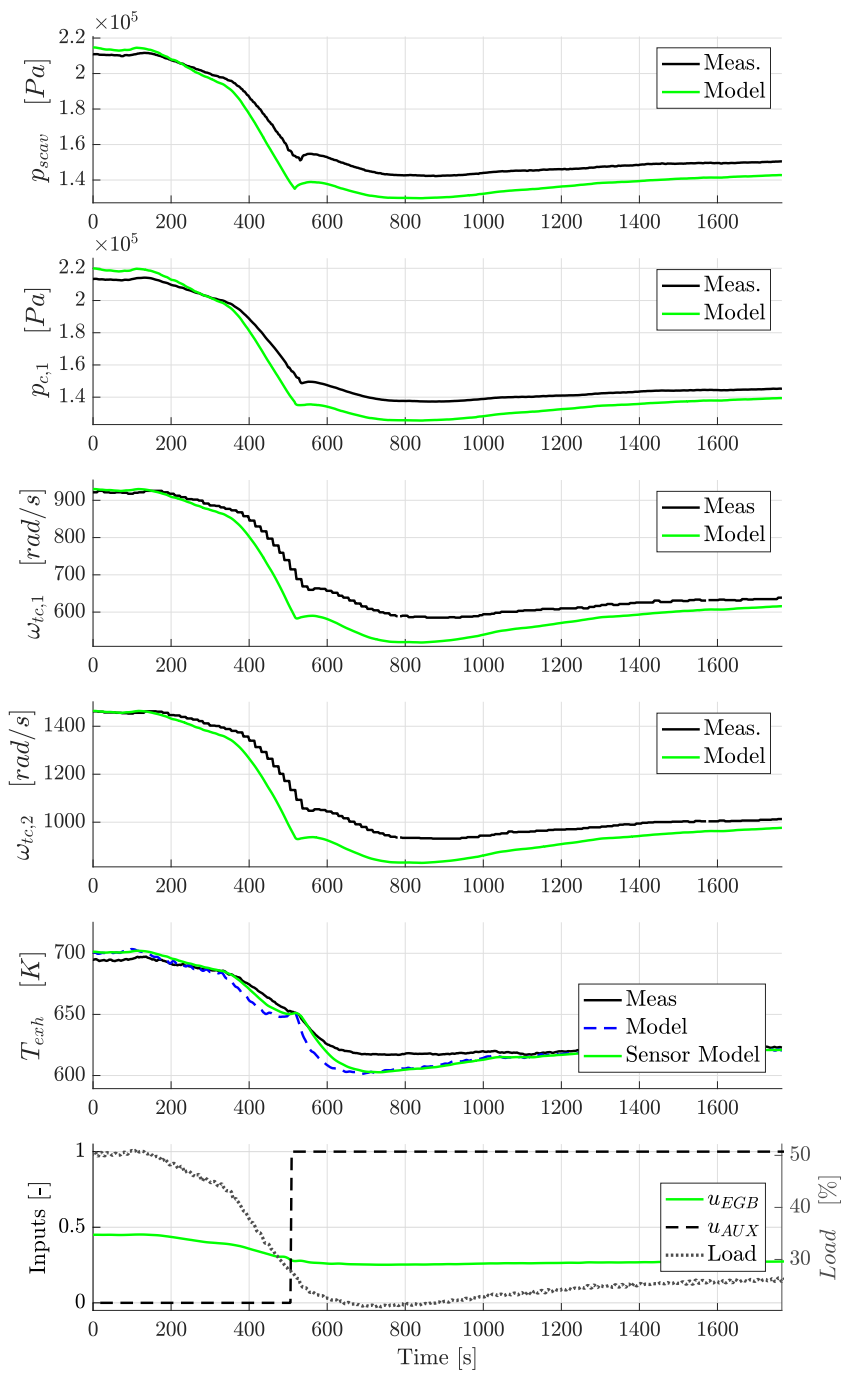

Figure 8. Model transient predictions and measured signals for a Load decrease scenario followed by an auxiliary blower start. The right axes in the bottom plot correspond to the percentage of engine load, depicted with a dotted line.

the latter having a larger stationary error. The temperature response is also faster than the measured, and peaks at a higher value. This introduces a longer transient until the modeled temperature settles at the stationary value, compared to the effects observed in the measured signal. The oxygen level goes back to atmospheric conditions after the EGR valve angle is closed, the agreement between the model and the measured signals is good.

\section{Guidelines for Future Engine Measurements}

Gathering sufficient measurement data at different operating points takes a lot of time since the measurement data is recorded while the ship is sailing. Hence, having guidelines 

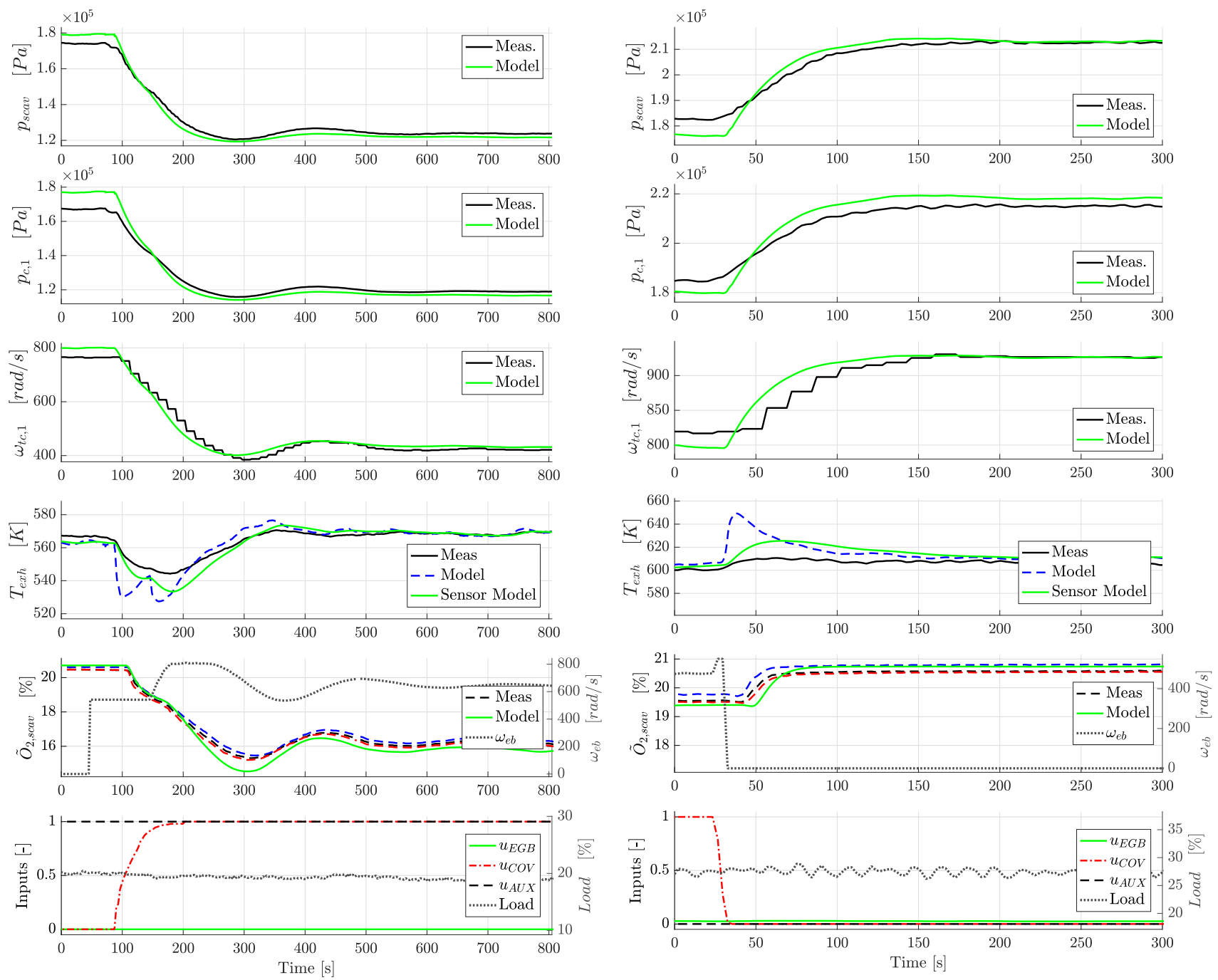

Figure 9. Model transient predictions and measured signals for an EGR start scenario. Note that the three measured Oxygen molar concentrations are plotted as dashed lines. In the same plot, the EGR blower speeds are shown as dotted lines with the corresponding axes on the right side.

Figure 10. Model transient predictions and measured signals for an EGR stop scenario. Note that the three measured Oxygen molar concentrations are plotted as dashed lines. In the same plot, the EGR blower speeds are shown as dotted lines with the corresponding axes on the right side.

of what measurements are important beforehand could be useful when parameterizing this model to other engines, and also to further improvements of the proposed engine model. The experience gained during the development of this engine model is summarized in this section.

First of all, fast temperature sensors mounted close to the cylinder exhaust valves would be very useful. Those measurements would help to validate the assumptions taken for the cylinder out temperature model described in Section 3.7. Moreover, those measurements together with fast measurements of the exhaust wall and the engine room temperature would permit to decouple the parameterization of the cylinder temperature model from the heat transfer model of the exhaust manifold.

Regarding the auxiliary blower, the engine designer does not choose of the specific blower that will be installed. Hence, the only information available are the two operating specifications that have to be fulfilled by the blower chosen by the engine manufacturer. Placing pressure sensors and the inlet and outlet of the blower as well as a blower speed measurement would be helpful for low load parameterization. In addition, it would be useful for improving the proposed dynamic model (14), by taking into consideration the speed dynamics of the blower.

Mass flow measurements could also be very helpful to validate the model extrapolation at low loads when the auxiliary blower is running, and the compressor is working well outside the mapped area. This measurement would 
be also helpful for validating the choice of a compressible restriction as the cylinder mass flow model. Furthermore, the parameterization process could be simplified, since the different submodels could be decoupled. Unfortunately, the installation of pressure based mass flow sensors is quite complicated since usually, they introduce too high-pressure drops.

\section{Propeller and Ship Resistance Models}

With the purpose to simulate the complete ship propulsion system, models for the propeller and the ship resistance are implemented. These models define two more states, the engine shaft rotational speed and the ship sailing speed. Moreover, they also provide the resistive load that the previously described two-stroke engine model has to overcome. The modeling follows the same steps as presented in Theotokatos and Tzelepis, ${ }^{38}$ which in turns follows the well-known modeling approach developed in Holtrop and Mennen. ${ }^{39}$

\subsection{Propeller and Shaft Models}

The ship propeller required torque, $Q_{P}$, and provided thrust, $T_{P}$, are computed using the definition of the nondimensional coefficients of torque and thrust, ${ }^{40} K_{Q}$ and $K_{T}$ respectively.

$$
\begin{gathered}
Q_{P}=K_{Q} \rho_{s w} N_{P}^{2} D_{P}^{5} \\
T_{P}=K_{T} \rho_{s w} N_{P}^{2} D_{P}^{4}
\end{gathered}
$$

The non-dimensional coefficients are computed with the help of the Wageningen B-Screw Series polynomials described in Van Lammeren et al. ${ }^{41}$ These polynomials are function of the propeller geometrical parameters, available from the propeller technical specifications, and the advance speed, $J_{A}$, which is computed as

$$
J_{A}=\frac{v_{A}}{D_{P} n_{P}}
$$

where $v_{A}$ is the advance speed of the propeller relative to the water, $D_{P}$ is the propeller diameter, and $n_{P}$ is the propeller speed in revolutions per second.

The shaft speed is calculated with the following differential equation

$$
\frac{d \omega_{e n g}}{d t}=\frac{Q_{\text {brake }}-Q_{P}}{J_{\text {eng }}+J_{\text {shaft }}+J_{P}+J_{\text {ent }}}
$$

where $Q_{\text {brake }}$ is the engine brake torque, and the different shaft system inertias are denoted as $J$ in the denominator. The entrained water inertia, $J_{e n t}$, is calculated using the regression model described in Parsons ${ }^{42}$ for Wageningen B-Series propellers.

\subsection{Ship Resistance Model}

With the produced propeller thrust and the calculated ship resistance, the current speed of the ship can be calculated as

$$
\frac{d v_{\text {ship }}}{d t}=\frac{T_{P}(1-t)-R_{\text {ship }}}{m_{\text {ship }}+m_{\text {hydro }}}
$$

where $t$ is the thrust deduction coefficient, $R_{\text {ship }}$ the ship resistance, $m_{\text {ship }}$ is the mass of the ship calculated with the displaced water volume and density. The added mass, $m_{\text {hydro }}$, represents the hydrodynamic inertial effects of the surrounding water. It is calculated using the linear equation proposed in Oltmann. ${ }^{43}$ The resistance and the thrust deduction coefficient are computed following the statistical prediction method described in Holtrop and Mennen, ${ }^{39}$ with the updated equations presented in Holtrop. ${ }^{44}$ The specific container ship geometrical characteristics are used in the calculation of $R_{\text {ship }}$.

The dynamic model formed by (55) and (56), is implemented in Simulink. Since not all hull design parameters required for calculating the ship resistance are known, the unknown values are adjusted, so the model agrees with the measurements. The measured engine braking power is used as input, and the calculated shaft rotational speed and vessel speed are plotted against the measured signals in Figure 11. As it can be seen, the model gives a reasonable estimate of the engine loading profile, while the vessel speed prediction is worse in general. It is important to mention that the model prediction could be improved by knowing more about the current loading of the vessel for each stationary point. Parameters like the draught and displacement depend on the amount of cargo and here are taken as constants to have an approximation for all the points shown in Figure 11. Moreover, the ship resistance is highly dependent on the sea conditions and the hull fouling level, ${ }^{45}$ and these conditions are unknown for the collected stationary points. Despite all these unknown parameters, this is a suitable starting point to simulate the complete container ship during transients. Moreover, it also enables to carry out simulations to study the effects of increased resistance based on sea conditions. ${ }^{38}$

\subsection{Engine Speed Governor}

The engine speed governor controls the fuel index value, $Y$, to match an ordered engine speed value. The governor is modeled as a PI controller, ${ }^{10}$ which in the frequency domain it is written as

$$
Y=K_{p} \Delta N_{e n g}+\frac{K_{i} \Delta N_{e n g}}{s}
$$

where $\Delta N_{\text {eng }}$ is the difference between the desired engine speed and the current engine speed. The parameters, $K_{p}$ and $K_{i}$ are the proportional and integral gains respectively. 

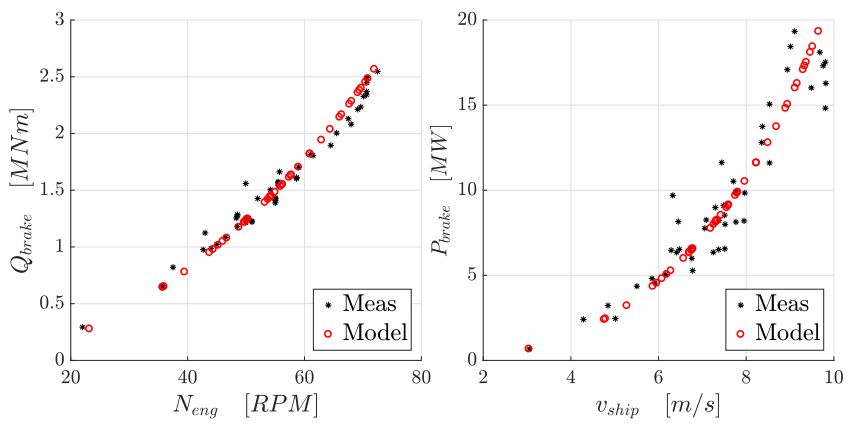

Figure 11. Propeller and ship resistance models vs measurements for the measured stationary operating points.

Figure 12 shows a simulation of the engine model together with the ship resistance model and the implemented engine speed governor. The ordered speed value is not available in the measurements, so in order to compare the ship speed dynamics, the measured engine speed is used as the governor input. As can be seen in the figure the model can predict reasonably well the measured necessary engine brake power based on the ordered fuel index. Note that a delay in the measured brake power can be observed, this is due to a delay in the measured engine torque signal. As can be seen in Figure 12 the measured engine speed and fuel index are synchronized with the model signals. On the other hand, the vessel speed prediction has a similar dynamic behavior as the measured signal despite the larger stationary difference. This difference is a consequence of the propeller and resistance model errors, as discussed in the previous section. The complete vessel model is simulated in Matlab Simulink, in a standard desktop computer, the simulation shown in Figure 12 is performed 55 times faster than real time.

\section{Conclusions}

An MVEM of a large two-stroke diesel engine with EGR is described and validated against measured data of a real engine operating in a sailing container ship. The required simulation time in a standard desktop computer is in general 50 times faster than real time. This fast simulation time makes the model suitable for the intended application of EGR and fuel controller development. A new analytic cylinder pressure model is also developed using cylinder pressure measurements from the real engine. The cylinder model is shown to be able to capture the effects of different control inputs to the prediction of engine power output and cylinder pressure.

The stationary parameterization procedure, necessary due to the low measurement data availability, manages to adjust the proposed MVEM response to the measured signals. The model is shown to capture the stationary engine operation for a wide span of engine loads well, from
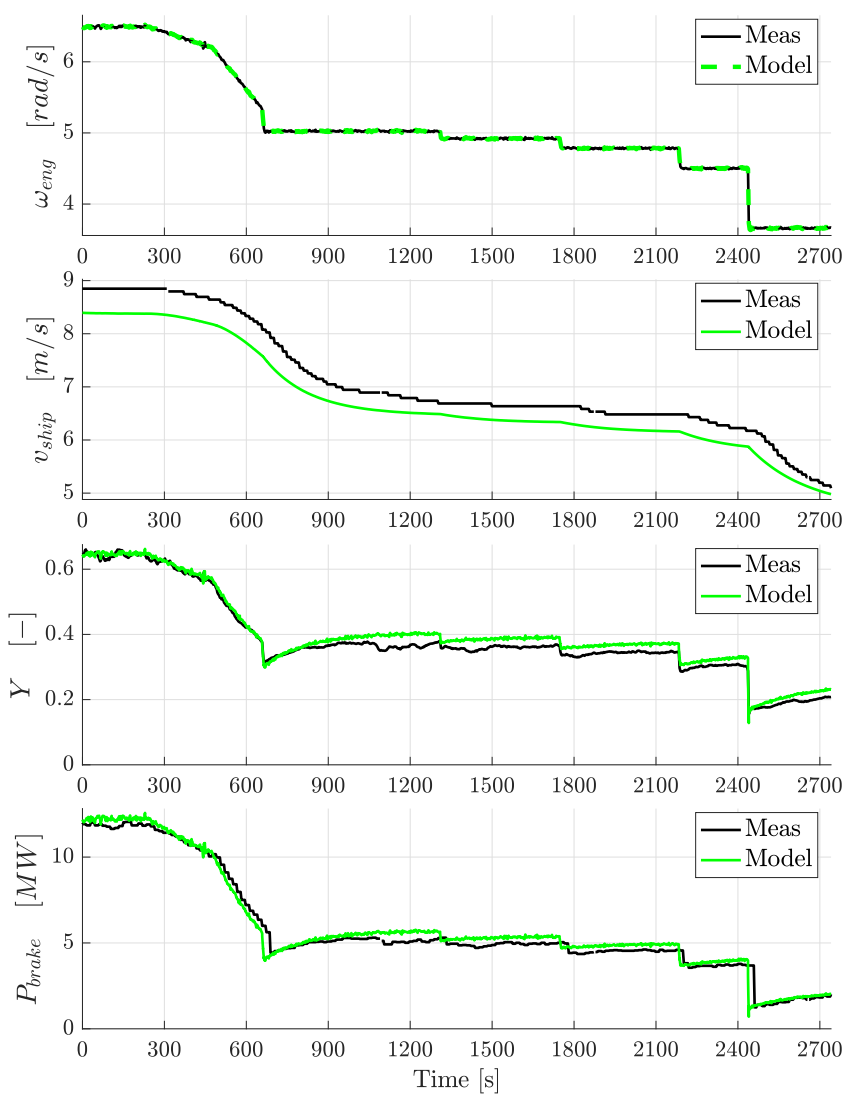

Figure 12. Comparison between the dynamic signals of the complete ship model with the speed governor and the measured signals.

$10 \%$ to $90 \%$, with and without the EGR system activated. The stationary relative errors are in general under $3.35 \%$ for both estimation and validation data which is a good indication of model accuracy.

To simplify the dynamic parameterization, the dynamic parameters are set to the nominal geometric values. The dynamic validation of the model shows that it is capable of following the measured engine signals during transients. However, the model transient response is for some cases faster than the measured, especially during the transients introduced when EGR is started and stopped. The applied solution is to increase the main turbocharger inertia by $30 \%$.

The use of control-oriented simulation models for designing and benchmarking EGR and fuel controllers will increase in the near future. Thus, the lessons learned during the complete model development project are summarized here for the research community. Essential signals to be included in future measurement campaigns and measurement setups for newly built engines are identified. These signals will improve the parameterization process of the proposed model and also help to validate further the model assumptions. 
Finally, models for the propeller and ship resistance are also investigated together with an engine speed governor. It is shown that these models can be used together with the proposed MVEM to simulate the complete propulsion system of container ships during maneuvering. This is of particular interest since the analysis of EGR controller performance during loading transients is one of the most important scenarios where the EGR control has to be improved. The main reason for this is that the more strict $N O_{x}$ emission limits are enforced in coastal areas where the ships need to be able to maneuver when approaching harbors.

\section{Acknowledgements}

MAN Diesel \& Turbo is greatly acknowledged for their support and discussions about the engine and for providing measurement data.

\section{Declaration of conflicting interests}

The authors declare that there is no conflict of interest.

\section{Funding}

This project has received funding from the European Union's Horizon 2020 research and innovation programme under grant agreement No 634135.

\section{References}

1. United Nations. Review of Maritime Transport. UN, 2016. ISBN 978-92-1-112904-5.

2. International Maritime Organization. MARPOL: Annex VI and NTC 2008, 2013: with Guidelines for Implementation. IMO, 2013. ISBN 978-92-801-15604.

3. Eriksson L and Nielsen L. Modeling and Control of Engines and Drivelines. John Wiley \& Sons, 2014.

4. Nielsen KV, Blanke M and Eriksson L. Adaptive observer for nonlinearly parameterized hammerstein system with sensor delay-applied to ship emissions reduction. IEEE Transactions on Control Systems Technology 2017; DOI:10. 1109/TCST.2017.2715004.

5. Nielsen KV, Blanke M, Eriksson L et al. Adaptive feedforward control of exhaust recirculation in large diesel engines. Control Engineering Practice 2017; 65: 26 - 35. DOI:10.1016/j.conengprac.2017.05.003.

6. Heywood JB. Internal Combustion Engine Fundamentals. McGraw-Hill, 1988.

7. Blanke $M$ and Anderson JA. On modelling large two stroke diesel engines: new results from identification. IFAC Proceedings Series 1985; : 2015-2020.

8. Woodward JB and Latorre RG. Modeling of diesel engine transient behavior in marine propulsion analysis. Transactions - Society of Naval Architects and Marine Engineers 1985; 92: 33 - 49.
9. Hendricks E. A compact comprehensive model of a large turbocharged two-stroke diesel engine. In SAE Technical Paper 861190.

10. Xiros N. Robust Control of Diesel Ship Propulsion. SpringerVerlag London, 2002.

11. Xiros NI and Theotokatos G. Improved transient control of a two-stroke marine diesel engine with variable geometry turbine. In ASME 2011 Internal Combustion Engine Division Fall Technical Conference, 2011. West Virginia, USA. DOI: 10.1115/ICEF2011-60199.

12. Theotokatos G. On the cycle mean value modelling of a large two-stroke marine diesel engine. Proc Inst Mech Eng M: Journal of engineering for the maritime environment 2010; 224(3): 193-206. DOI:10.1243/14750902JEME188.

13. Guan C, Theotokatos G, Zhou P et al. Computational investigation of a large containership propulsion engine operation at slow steaming conditions. Appl Energy 2014; 130: 370 - 383. DOI:10.1016/j.apenergy.2014.05.063.

14. Guan C, Theotokatos G and Chen H. Analysis of two stroke marine diesel engine operation including turbocharger cutout by using a zero-dimensional model. Energies 2015; 8(6): 5738 - 5764. DOI:10.3390/en8065738.

15. Theotokatos G, Guan C, Chen H et al. Development of an extended mean value engine model for predicting the marine two-stroke engine operation at varying settings. Energy 2018; 143: 533 - 545. DOI:10.1016/j.energy.2017.10.138.

16. Malkhede DN, Seth B and Dhariwal H. Mean value model and control of a marine turbocharged diesel engine. In $S A E$ Technical Paper 2005-01-3889. DOI:10.4271/2005-01-3889.

17. Baldi F, Theotokatos G and Andersson K. Development of a combined mean valuezero dimensional model and application for a large marine four-stroke diesel engine simulation. Appl Energy 2015; 154: 402 - 415. DOI:10.1016/j.apenergy.2015. 05.024 .

18. Hansen JM, Zander CG, Pedersen N et al. Modelling for control of exhaust gas recirculation on large diesel engines. 9th IFAC Conference on Control Applications in Marine Systems 2013; DOI:10.3182/20130918-4-JP-3022.00013.

19. Wahlström $J$ and Eriksson L. Modelling diesel engines with a variable-geometry turbocharger and exhaust gas recirculation by optimization of model parameters for capturing nonlinear system dynamics. Proceedings of the Institution of Mechanical Engineers, Part D, Journal of Automobile Engineering 2011; 225(7): 960-986.

20. Alegret G, Llamas X, Vejlgaard-Laursen M et al. Modeling of a large marine two-stroke diesel engine with cylinder bypass valve and EGR system. IFAC-PapersOnLine 2015; 48(16): 273 - 278. DOI:10.1016/j.ifacol.2015.10.292. 10th IFAC Conference on Manoeuvring and Control of Marine Craft.

21. Llamas X and Eriksson L. A model of a marine two-stroke diesel engine with EGR for low load simulation. In 9th EUROSIM Congress on Modelling and Simulation, 2016. 
22. Nielsen KV, Blanke M, Eriksson L et al. Controloriented model of molar scavenge oxygen fraction for exhaust recirculation in large diesel engines. ASME J Dyn Sys, Meas, Control 2016; 139(2). DOI:10.1115/1.4034750.

23. Wang Z, Zhou S, Feng Y et al. Research of NOx reduction on a low-speed two-stroke marine diesel engine by using EGR (exhaust gas recirculation) - CB (cylinder bypass) and EGB (exhaust gas bypass). International Journal of Hydrogen Energy 2017; 42(30): 19337 - 19345. DOI:10. 1016/j.ijhydene.2017.06.009.

24. Wang Z, Zhou S, Feng $\mathrm{Y}$ et al. Investigation of EGR with EGB (exhaust gas bypass) on low speed marine diesel engine performance and emission characteristics. In ASME International Conference on Offshore Mechanics and Arctic Engineering. Trondheim, Norway. DOI:10.1115/ OMAE2017-62606.

25. McBride BJ, Zehe MJ and Gordon S. NASA Glenn coefficients for calculating thermodynamic properties of individual species. NASA/TP-2002-211556 2000; .

26. Llamas $X$ and Eriksson L. Control-oriented compressor model with adiabatic efficiency extrapolation. SAE International Journal of Engines 2017; 10(4).

27. Llamas $X$ and Eriksson L. Parameterizing compact and extensible compressor models using orthogonal distance minimization. ASME J Gas Turb Pwr 2017; 139(1). DOI: 10.1115/1.4034152.

28. Çengel YA and Boles MA. Thermodynamics. 6th ed. New York, US: McGraw-Hill Higher Education, 2007.

29. Eriksson L. Modeling and control of turbocharged SI and DI engines. Oil \& Gas Science and Technology 2007; 62(4): 523-538. DOI:10.2516/ogst:2007042.

30. Eriksson L and Andersson I. An analytic model for cylinder pressure in a four stroke SI engine. In SAE Technical Paper 2002-01-0371. DOI:10.4271/2002-01-0371.

31. Andersen FH. Integrated Analysis of the Scavenging Process in Marine Two-Stroke Diesel Engines. PhD Thesis, Technical University of Denmark, 2015.

32. Sigurdsson E, Ingvorsen $\mathrm{K}$, Jensen $\mathrm{M}$ et al. Numerical analysis of the scavenge flow and convective heat transfer in large two-stroke marine diesel engines. Appl Energy 2014; 123: 37 - 46. DOI:10.1016/j.apenergy.2014.02.036.

33. Lamas MI and Rodíguez Vidal CG. Computational fluid dynamics analysis of the scavenging process in the MAN B\&W 7S50MC two-stroke marine diesel engine. Journal of Ship Research - Society of Naval Architects and Marine Engineers 2012; 56(3): 154-161. DOI:doi:10.5957/JOSR.56. 3.120001 .

34. Merker GP and Gerstle M. Evaluation on two stroke engines scavenging models. In SAE Technical Paper 970358. DOI: $10.4271 / 970358$.

35. Kavtaradze RZ, Zeilinger $\mathrm{K}$ and Zitzler G. Ignition delay in a diesel engine utilizing different fuels. High Temperature 2005; 43(6): 951-960. DOI:10.1007/s10740-005-0143-z.
36. Flagan RC and Seinfeld JH. Fundamentals of air pollution engineering. Prentice-Hall, Inc., 1988. ISBN 0-13-332537-7.

37. Eriksson L. Mean value models for exhaust system temperatures. In SAE Technical Paper 2002-01-0374. DOI: 10.4271/2002-01-0374.

38. Theotokatos G and Tzelepis V. A computational study on the performance and emission parameters mapping of a ship propulsion system. Proc Inst Mech Eng M: Journal of engineering for the maritime environment 2015; 229(1): 5876. DOI:10.1177/1475090213498715.

39. Holtrop J and Mennen G. An approximate power prediction method. International Shipbuilding Progress 1982; 29(335): 166-170.

40. Carlton J. Marine Propellers and Propulsion. 3rd ed. Butterworth-Heinemann, 2012.

41. van Lammeren WPA, van Manen JD and Oosterveld MWC. The Wageningen B-Screw Series. Transactions SNAME 1969; .

42. Parsons MG. Mode coupling in torsional and longitudinal shafting vibrations. Marine Technology 1983; 20(3).

43. Oltmann P. Identification of hyrodynamic damping derivatives - a pragmatic approach. In Proceedings of the international conference on marine simulation and ship manoeuverability (MARSIM'03). Kanazawa, Japan.

44. Holtrop J. A statistical re-analysis of resistance and propulsion data. International Shipbuilding Progress 1984; 31(363): 272-276.

45. MAN Diesel \& Turbo. Basic principles of ship propulsion, 2012.

\section{Appendix I}

\section{Nomenclature}

$\begin{array}{ll}\dot{Q} & \text { Heat transfer } \\ \tilde{O}_{2} & \text { Oxygen Molar Fraction } \\ \tilde{X} & \text { Molar Fraction } \\ A & \text { Area or Equivalent Area } \\ D & \text { Diameter } \\ J & \text { Inertia or propeller advance speed } \\ M & \text { Molar mass } \\ N & \text { Rotational Speed in [rpm] } \\ P & \text { Power } \\ Q & \text { Torque } \\ R & \text { Gas Constant or Resistance force } \\ R H & \text { Relative Humidity } \\ T & \text { Temperature or Thrust } \\ V & \text { Volume } \\ W & \text { Mass Flow } \\ X & \text { Mass Fraction }\end{array}$




$\begin{array}{ll}Y & \text { Fuel Index } \\ C F D & \text { Computational Fluid Dynamics } \\ E G R & \text { Exhaust Gas Recirculation } \\ E R M & \text { Engine Running Mode } \\ E V C & \text { Exhaust Valve Closing } \\ E V O & \text { Exhaust Valve Opening } \\ I P C & \text { Intake Port Closing } \\ I P O & \text { Intake Port Opening } \\ M V E M & \text { Mean Value Engine Model } \\ N E C A & \text { NOx Emission Control Area } \\ R M S & \text { Root-mean-square error } \\ c_{p} & \text { Specific heat at constant pressure } \\ c_{v} & \text { Specific heat at constant volume } \\ e & \text { Relative error } \\ h & \text { Heat Transfer coefficient } \\ k & \text { Model parameter } \\ m & \text { Mass } \\ n & \text { Rotational Speed in [rps] } \\ p & \text { Pressure } \\ u & \text { Thrust deduction coefficient } \\ v & \text { Control input } \\ \text { Velocity }\end{array}$

\section{Greek Symbols}

$\begin{array}{ll}\alpha & \text { Crank Angle (control input) } \\ \epsilon & \text { Emissivity } \\ \eta & \text { Efficiency } \\ \gamma & \text { Specific heats ratio } \\ \Omega & \text { Work } \\ \omega & \text { Rotational Speed in }[\mathrm{rad} / \mathrm{s}] \\ \Phi & \text { Flow coefficient } \\ \Pi & \text { Pressure ratio } \\ \Psi & \text { Energy Transfer coefficient } \\ \rho & \text { Density } \\ \sigma & \text { Boltzmann constant } \\ \theta & \text { Crank Angle } \\ \varphi & \text { Vector of parameters }\end{array}$

\section{Subscripts}

$\begin{array}{ll}A & \text { Advance } \\ a c & \text { Air Cooler } \\ a m b & \text { Ambient } \\ A u x & \text { Auxiliary Blower } \\ c & \text { Compressor }\end{array}$

\begin{tabular}{|c|c|}
\hline$c o$ & Compression \\
\hline comb & Combustion \\
\hline$C O V$ & Cut-Out Valve \\
\hline$c v$ & Convection \\
\hline$c y l$ & Cylinder \\
\hline del & Delivered \\
\hline$e$ & External \\
\hline$e b$ & EGR Blower \\
\hline$e f f$ & Effective \\
\hline$E G B$ & Exhaust Gas Bypass \\
\hline$e n g$ & Engine \\
\hline ent & Entrained water \\
\hline$e w$ & Exhaust wall \\
\hline$e x$ & Expansion \\
\hline$e x h$ & Exhaust \\
\hline$f$ & Fuel \\
\hline$i$ & Internal \\
\hline in & Inlet \\
\hline$i n j$ & Injection \\
\hline meas & Measured \\
\hline mech & Mechanical \\
\hline $\operatorname{mix}$ & Gas Mixer \\
\hline $\bmod$ & Model \\
\hline out & Outlet \\
\hline$P$ & Propeller \\
\hline scav & Scavenging \\
\hline$S D V$ & Shut Down Valve \\
\hline sen & Sensor \\
\hline shaft & Engine crankshaft \\
\hline ship & Ship \\
\hline$s w$ & Sea water \\
\hline$t$ & Turbine \\
\hline$t c$ & Turbocharger \\
\hline$v$ & Valve \\
\hline
\end{tabular}

\section{Appendix II: Derivatives of} thermodynamical properties

The working fluid is assumed to be a thermally perfect gas formed by a mixture of different gases. Hence, when deriving the expressions for the control volume dynamics described in Section 3.2, partial derivatives of the thermodynamical properties appear. This Appendix briefly 
introduces how these expressions are computed based on the NASA polynomials ${ }^{25}$ for the considered expressions.

The molar mass and the gas constant of a mixture of $N$ species is computed as in Çengel and Boles ${ }^{28}$

$$
\begin{aligned}
& M(X)=\frac{\sum_{j=1}^{N} X_{j}}{\sum_{j=1}^{N} X_{j} / M_{j}} \\
& R(X)=\frac{\tilde{R}}{M(X)}
\end{aligned}
$$

The derivative of the gas constant with respect to the mass fractions is computed as

$$
\frac{d R(X)}{d X}=\frac{-\tilde{R}}{M(X)^{2}} \nabla_{x} M(X)=-\tilde{R}\left(\begin{array}{c}
\vdots \\
\frac{1}{M(X)}-\frac{1}{M_{i}} \\
\vdots
\end{array}\right)
$$

It is possible to transform from molar to mass fractions and vice-versa using the following equations

$$
X_{i}=\frac{\tilde{X}_{i} M_{i}}{M} \quad \tilde{X}_{i}=\frac{X_{i} M}{M_{i}}
$$

for a given gas $i$ in the mixture.

With the definition of the NASA polynomials, the specific heat at constant pressure of gas $i$ in the mixture is computed as

$$
c_{p, i}(T)=\frac{\tilde{R}}{M_{i}} \underbrace{\left(a_{i, 1}+a_{i, 2} T+a_{i, 3} T^{2}+a_{i, 4} T^{3}+a_{i, 5} T^{4}\right)}_{P_{n, i}(T)}
$$

where $a_{i, x}$ are the coefficients of the NASA polynomial, ${ }^{25}$ $P_{n, i}(T)$, for a given gas in the mixture, $i$. Considering the gas as a nonreacting mixture, the specific heat at constant pressure can be computed as the sum of the contributions of each mixture component ${ }^{28}$

$$
c_{p}(X, T)=\sum_{j=1}^{N} X_{j} c_{p, j}(T)
$$

The specific heat at constant volume can be computed from the mixture gas constant and the specific heat at constant pressure as follows since an ideal gas is assumed ${ }^{28}$

$$
c_{v}(X, T)=c_{p}(X, T)-R(X)
$$

The partial derivatives of the specific heat at constant volume appear when differentiating the energy equation in (4a). They can be computed from the previous equation using the chain rule

$$
\begin{aligned}
& \frac{\partial c_{v}(X, T)}{\partial X}=\frac{\partial c_{p}(X, T)}{\partial X}-\frac{d R(X)}{d X} \\
& \frac{\partial c_{v}(X, T)}{\partial T}=\frac{\partial c_{p}(X, T)}{\partial T}
\end{aligned}
$$

The required partial derivatives of the specific heat at constant pressure can be computed as

$$
\begin{aligned}
& \frac{\partial c_{p}(X, T)}{\partial X}=\nabla_{x} c_{p}(X, T)=\left(\begin{array}{c}
\vdots \\
\frac{\tilde{R}}{M_{i}} P_{n, i}(T) \\
\vdots
\end{array}\right) \\
& \frac{\partial c_{p}(X, T)}{\partial T}=\sum_{j=1}^{N} \frac{X_{j} \tilde{R}}{M_{j}} \frac{d P_{n, i}(T)}{d T}
\end{aligned}
$$

where $\frac{d P_{n, i}(T)}{d T}$ is the derivative with respect to the control volume temperature of the NASA polynomial of the arbitrary gas $i$ in the mixture. 